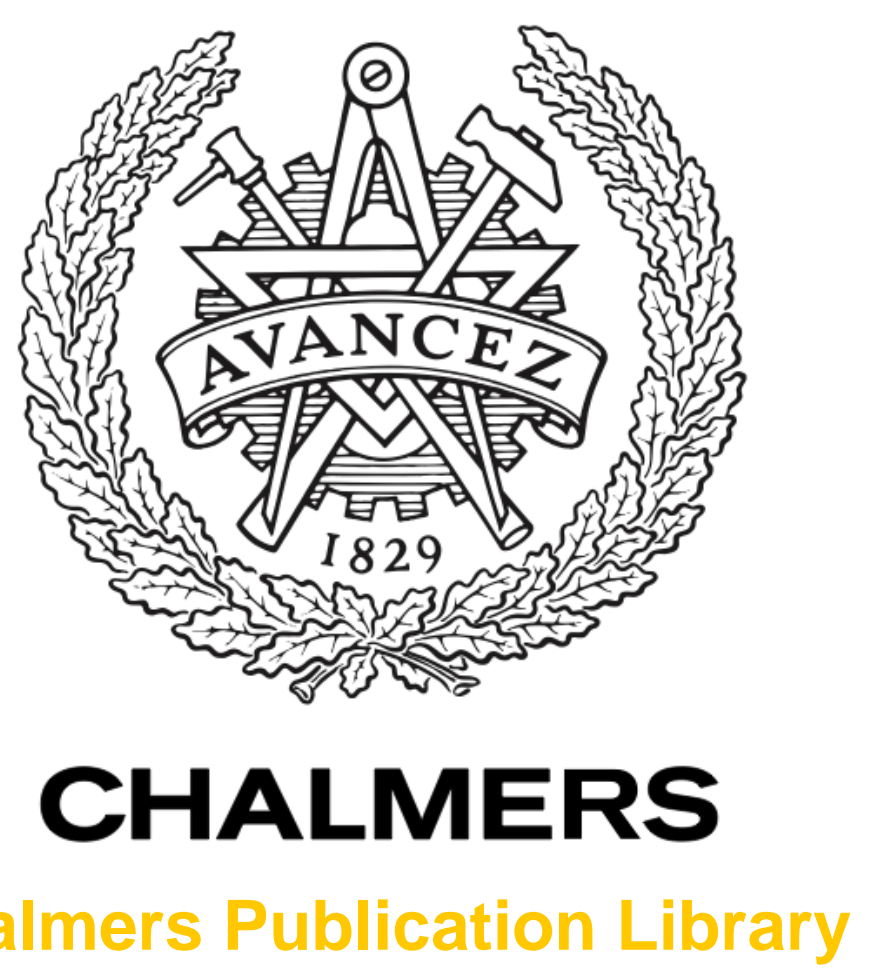

Challmers Publication Library

\title{
Multitarget Sensor Resolution Model and Joint Probabilistic Data Association
}

This document has been downloaded from Chalmers Publication Library (CPL). It is the author's version of a work that was accepted for publication in:

IEEE Transactions on Aerospace and Electronic Systems (ISSN: 0018-9251)

Citation for the published paper:

Svensson, D. ; Ulmke, M. ; Hammarstrand, L. (2012) "Multitarget Sensor Resolution Model and Joint Probabilistic Data Association". IEEE Transactions on Aerospace and Electronic Systems, vol. 48(4), pp. 3418-3434.

http://dx.doi.org/10.1109/TAES.2012.6324722

Downloaded from: http://publications.lib.chalmers.se/publication/165768

Notice: Changes introduced as a result of publishing processes such as copy-editing and formatting may not be reflected in this document. For a definitive version of this work, please refer to the published source. Please note that access to the published version might require a subscription.

Chalmers Publication Library (CPL) offers the possibility of retrieving research publications produced at Chalmers University of Technology. It covers all types of publications: articles, dissertations, licentiate theses, masters theses, conference papers, reports etc. Since 2006 it is the official tool for Chalmers official publication statistics. To ensure that Chalmers research results are disseminated as widely as possible, an Open Access Policy has been adopted.

The CPL service is administrated and maintained by Chalmers Library. 


\title{
Multitarget Sensor Resolution Model and Joint Probabilistic Data Association
}

\author{
Daniel Svensson, Martin Ulmke, and Lars Hammarstrand
}

\begin{abstract}
In the design of target tracking algorithms, the aspect of sensor resolution is rarely considered. Instead, it is usually assumed that all targets are always resolved, and that the only uncertainties in the data association are which targets that are detected, and which measurement each detected target gave rise to. However, in situations where the targets are closely spaced in relation to the sensor resolution, this assumption is not valid, and may lead to degraded tracking performance due to an incorrect description of the data. In this paper, we present a framework for handling sensor resolution effects for an arbitrary, but known, number of targets. We propose a complete multitarget sensor resolution model that can be incorporated into traditional Bayesian tracking filters. Further, the exact form of the posterior probability density function is derived, and two alternative ways of approximating that exact posterior density with a Joint Probabilistic Data Association (JPDA) filter are proposed. Evaluations of the resulting filters on simulated radar data show significantly increased tracking performance compared to the JPDA filter without a resolution model.
\end{abstract}

\section{Index Terms}

Target tracking, state estimation, Bayes methods, sensor models, radar detection, radar resolution.

D. Svensson is with the Department of Signals and Systems, Chalmers University of Technology, SE-41296 Gothenburg, Sweden, E-mail: daniel.svensson@chalmers.se, and with Electronic Defence Systems, Saab AB, SE-412 89 Gothenburg, Sweden, E-mail: daniel.x.svensson@saabgroup.com.

M. Ulmke is with the Department of Sensor Data and Information Fusion, Fraunhofer-FKIE, Wachtberg, Germany, E-mail: martin.ulmke@fkie.fraunhofer.de.

L. Hammarstrand (previously Danielsson) is with the Department of Signals and Systems, Chalmers University of Technology, SE-41296 Gothenburg, Sweden, E-mail: lars.hammarstrand@chalmers.se. 


\section{INTRODUCTION}

Traditionally the aspect of sensor resolution has not been considered in the design of target tracking algorithms [2]. Instead, it has been assumed that the targets are always resolved, and that the data association problem only regards the assignment of measurements to single targets. In many situations, that assumption is reasonable, but there are important cases when the resolution limitations of the sensor cannot be ignored [10]. Such cases arise when targets are temporarily closely spaced in relation to the resolution of the sensor, e.g., when tracking aircraft in formation, or in convoy tracking for ground surveillance. For such applications, ignoring the limited resolution of the sensors may lead to an incorrect interpretation of the data, which results in degraded performance, in particular due to premature deletion of tracks.

To account for resolution in a tracking algorithm, the resolution phenomena must be modeled. One way of modeling them is to express the capability of the sensor to resolve individual targets in a group by a resolution probability, and to have a model for the merged measurement that arises when the targets are unresolved. Two approaches have been proposed in the literature which follow this line of modeling. In [8], a grid-based resolution model for two targets is proposed, where the resolution probability is zero if the targets are within the same resolution cell, and one otherwise. The integration into the Joint Probabilistic Data Association (JPDA) filter [13] is also described, and in [19] the model is further extended to a Multiple Hypothesis Tracking (MHT) framework [1], [2], [11], [20]. Even though a fixed grid often is a good approximation of the signal processing procedure, the capability of a sensor to resolve targets generally depends on their positions relative to the sensor. Hence, in [18], a simple, but qualitatively correct, resolution model that take relative positions into account is proposed. Further, its incorporation into an MHT filter is also presented. In [5], the model in [18] is combined with the socalled descriptor system approach, which results in resolution-model extensions of the Joint Interacting Multiple Model Coupled Probabilistic Data Association (JIMMCPDA) filter, and its track-coalescenceavoiding version, the JIMMCPDA* filter [3]. An exact Bayesian filter for two targets is also developed. Further, in [6], the hybrid sequential importance resampling particle filter [4] is extended with the above two-target resolution model. However, just as the resolution model in [8], the model in [18], and the filters based upon it, are restricted to only consider two targets. An alternative approach to the tracking of closely spaced objects is to treat them as a group. For an overview on group target tracking, see [28]. A benchmark scenario for tracking including (two) closely spaced targets is given in [27], and a solution to it based on angle estimation of two unresolved targets [22] (with extension in [26]) is presented in [23]. 
In this article, we consider the modeling of limited sensor resolution for an arbitrary, but known, number of targets. It is hence the first approach to consider resolution problems for more than two closely-spaced targets. The main contribution of the article is a framework for handling resolution limitations, which can be easily incorporated into a Bayesian tracking setting. The framework relies on a graph description of a resolution event, and on modeling the resolution probability as independent between target pairs. To complete the framework and to attain a multitarget resolution model, a model for the resolution probability for two targets and a group measurement model for an arbitrary number of targets are needed; for example, the models in [8] or [18]. Preliminary results have previously been published in [24], [25].

The outline of the article as follows. In Section II, the problem formulation is stated. The proposal of a framework for sensor resolution modeling is given in Section III, together with the graph description of a resolution event. To complete the framework, specific measurement and resolution models for radar sensors are suggested in Section IV. In Section V, the exact calculation of the posterior pdf under both resolution and data association conflicts is described, while a Gaussian-mixture approximation is presented in Section VI. In Section VII, two alternative approaches of incorporating the resolution model into the JPDA filter are presented, and in Section VIII those approaches are evaluated on simulated radar data. In the evaluation, the tracking performance is compared to that of the JPDA filter without a resolution model. The results show improved performance for all considered setups. Finally, in Section IX, conclusions are drawn.

\section{PROBLEM FORMULATION}

The general problem considered in this article is tracking of a known number of targets under resolution limitations and unknown data associations. To this problem there are several subproblems. First, to model the probability that a group of targets is unresolved; second, to model the corresponding merged group measurement; and third, to find how these models can be incorporated into a general tracking framework.

The kinematic states of the $N$ targets are represented by a joint state vector

$$
\mathbf{x}_{k}=\left[\begin{array}{llll}
\left(\mathbf{x}_{k}^{(1)}\right)^{T} & \left(\mathbf{x}_{k}^{(2)}\right)^{T} & \ldots & \left(\mathbf{x}_{k}^{(N)}\right)^{T}
\end{array}\right]^{T},
$$

where $\mathbf{x}_{k}^{(i)}$ is the state of target $i$. At each time instant, $t_{k}$, a sensor produces measurements $\mathbf{Z}_{k}$ which contain information regarding the kinematic states $\mathbf{x}_{k}$. The collection of measurements from discrete time index 1 to index $k$ is represented by

$$
\mathbf{Z}^{k}=\left\{\mathbf{Z}_{1}, \mathbf{Z}_{2}, \ldots, \mathbf{Z}_{k}\right\}
$$


The goal of a tracking filter is to recursively calculate the posterior density $p\left(\mathbf{x}_{k} \mid \mathbf{Z}^{k}\right)$, from which optimal state estimates can be derived. To be able to calculate the density, models for the sensor measurements and for the motion of the targets are necessary. Additionally, when limited sensor resolution is considered, a model that describes that phenomenon is also needed. In the following, the considered process and measurement models are presented, while radar resolution models are proposed and discussed in Sections III and IV.

\section{A. Process model}

The process model, often called the motion model, describes the dynamics of the targets. Given the state $\mathbf{x}_{k-1}$ at time index $k-1$, the model describes the evolution of the state up to time index $k$. For the general case,

$$
\mathbf{x}_{k}=f_{k-1}\left(\mathbf{x}_{k-1}, \mathbf{v}_{k-1}\right)
$$

where $f_{k-1}$ is the system function describing the transition from time $k-1$ to time $k$, and $\mathbf{v}_{k-1}$ is a process noise that describes the uncertainties in the prediction. For Gauss-Markov systems, which we mainly consider in this article, it holds that

$$
\mathbf{x}_{k}=\mathbf{F}_{k-1} \mathbf{x}_{k-1}+\mathbf{v}_{k}
$$

where $\mathbf{F}_{k-1}$ is the multi-target system matrix at time $k-1$, and $\mathbf{v}_{k}$ is Gaussian distributed with zero mean and covariance matrix $\mathbf{Q}_{k}$.

\section{B. Sensor model}

The sensor model describes the relation between the received measurements and the target states. The measurements received at a certain time index include both target-generated detections and spurious measurements that are due to false alarms and clutter (henceforth subsumed as clutter).

The joint measurement vector at time $k$ is given by

$$
\mathbf{z}_{k}=\left[\begin{array}{llll}
\left(\mathbf{z}_{k}^{(1)}\right)^{T} & \left(\mathbf{z}_{k}^{(2)}\right)^{T} & \ldots & \left(\mathbf{z}_{k}^{\left(M_{k}\right)}\right)^{T}
\end{array}\right]^{T} .
$$

The heritage of the data is unknown, which means that it is not known which $M_{k, c}$ measurements are clutter and which $M_{k, t}$ measurements are target-generated. Additionally, it is not known which target or targets gave rise to each of the true detections. 
The uncertainty in the discrimination between clutter and target-generated measurements is mathematically described as

$$
\mathbf{Z}_{k}=\left(\boldsymbol{\Pi}_{p} \otimes \mathbf{I}_{N_{z} \times N_{z}}\right)\left[\begin{array}{l}
\mathbf{Z}_{k}^{c} \\
\mathbf{Z}_{k}^{t}
\end{array}\right],
$$

where $\mathbf{Z}_{k}^{c}$ and $\mathbf{Z}_{k}^{t}$ are ordered vectors of clutter- and target-originated measurements, respectively, $N_{z}$ is the dimension of the single-measurement space, and $\otimes$ denotes the Kronecker product. Further, $\Pi_{p}$ is an $M_{k}$-dimensional random permutation matrix which models the uncertainty with respect to which measurements are generated by targets. ${ }^{1}$

The uncertainty in the association between target-generated measurements and true targets, and the model for the corresponding target-generated measurements, are here described as

$$
\mathbf{Z}_{k}^{t}=\left(\mathbf{C}_{k} \otimes \mathbf{I}_{N_{z} \times N_{z}}\right) h_{k}\left(\mathbf{x}_{k}\right)+\mathbf{w}_{k},
$$

where $\mathbf{C}_{k}$ is an unknown $M_{k, t} \times N$-dimensional matrix which determines the contribution of each target on each target-generated measurement. When sensor resolution problems are not present, a measurement can only originate from a single target. In that case, $\mathbf{C}_{k}$ has at most one single non-zero element per row (a one), which determines the originating target of that measurement. However, for situations with resolution limitations, several targets can be perceived as one by the sensor, and thus give rise to a joint/merged measurement. Then, the $\mathbf{C}_{k}$ matrix will have several non-zero elements in the corresponding row, where the size of each element describes the contribution from each target to that measurement. ${ }^{2}$ The multitarget observation function $h_{k}$ transforms the joint state vector $\mathbf{x}_{k}$ to the measurement space, and the noise process $\mathbf{w}_{k}$ is assumed Gaussian with zero mean and block-diagonal covariance matrix $\mathbf{R}_{k}$. For linear measurement models, the target-generated measurements are given by

$$
\mathbf{Z}_{k}^{t}=\left(\mathbf{C}_{k} \otimes \mathbf{I}_{N_{z} \times N_{z}}\right) \tilde{\mathbf{H}} \mathbf{x}_{k}+\mathbf{w}_{k}
$$

where

$$
\tilde{\mathbf{H}}=\operatorname{diag}\{\underbrace{\mathbf{H}, \cdots, \mathbf{H}}_{N \text { times }}\},
$$

and where $\mathbf{H}$ is the single-target observation matrix. The measurement noise $\mathbf{w}_{k}$ is assumed independent of the process noise $\mathbf{v}_{k}$.

\footnotetext{
${ }^{1}$ This matrix is equivalent to the $\tilde{\chi}_{t}$ matrix in [5, p. 615].

${ }^{2}$ Comparing with the descriptor system in [5, p. 615], $\mathbf{C}_{k}$ corresponds to the operator $\Phi\left(\phi^{\prime}\right)$.
} 
For the measurement model to be complete, we also need to model the properties of the clutter measurements. We here assume a spatially homogeneous Possion process. A single clutter measurement, $\mathbf{z}_{k}^{c}$, is hence distributed as

$$
\mathbf{z}_{k}^{c} \sim \operatorname{Uniform}(\mathrm{FoV})
$$

where FoV is the field-of-view of the sensor, while the total number of clutter detections, $M_{k, c}$, is distributed according to

$$
M_{k, c} \sim \operatorname{Poisson}(\lambda \cdot|\mathrm{FoV}|)
$$

in which $\lambda$ is the clutter intensity and $\mid$ FoV $\mid$ the volume of the FoV. The algorithms described in this article, however, are not restricted to Poisson-distributed clutter.

\section{SENSOR RESOLUTION MODELING FRAMEWORK FOR ARBITRARY TARGET NUMBERS}

To be able to track targets under resolution limitations with high accuracy, we wish to create a model that

1) represents the probability that a group of targets is unresolved in a qualitatively correct way,

2) provides a reasonable description of the properties of a measurement from a group, and

3) is mathematically tractable and possible to incorporate in a Bayesian tracking framework.

In this section, we propose a novel framework for sensor resolution modeling for arbitrary, but known, number of targets, which fulfills the three requirements above. To complete the framework, models for the resolution probability of two targets and a measurement model for a group target are required. In Section IV, two such models for radar sensors are presented.

We start by defining a group.

Definition 1: A group is defined as a set of targets that upon detection by the sensor gives rise to a single (joint) measurement.

Thus, a group is not equivalent to a set of targets that move in a coordinated fashion, but a single resolved target, or a set of more than one targets, which at a certain instant of time are so closely spaced that they are not resolvable by the sensor. Note that the definition is on a per-scan basis, so that groups can be formed and dissolved from scan to scan. Also note that the current article does not address multi-sensor scenarios, but should such systems be of interest, the sensors would have to be considered in separate filter recursions. 
At a certain time instant, there are many different possibilities regarding which targets form a group and which are resolved. This is similar to the classic data association problem, where there are many different explanations regarding measurement-to-target associations. We thus make the following definition of a resolution event:

Definition 2: A resolution event is defined as a partition of the set of all targets into groups.

That means, the set of all targets is divided into a set of non-empty non-overlapping subsets.

Example 1: Say that there are five targets present in the scene. An example of a resolution event, $\mathcal{R}$,

is then: $\mathcal{R}=\{\{1,2\},\{3\},\{4,5\}\}$, meaning that one group is formed by the set of targets 1 and 2 , one group is formed by the single-object set of target 3, and a third group is formed by targets 4 and 5 .

So, at a given time instant, many resolution events are possible, and we need a resolution model to provide information regarding the probability of each such event.

\section{A. Resolution probabilities}

To model a resolution event, we propose the use of a graph representation, where each node in the graph represents a target, and where an edge between nodes represents the event that those two targets are mutually unresolved. A group is thus described by a connected sub-graph, i.e., a sub-graph with all nodes being connected. We make the following definition.

Definition 3: A resolution graph is one possible representation of a resolution event. In the graph, the nodes are defined by the target states, and a pair of unresolved targets is represented by a symmetric link between the respective nodes of the graph.

As the definition implies, a resolution event (cf. Definition 2) may correspond to several resolution graphs. For an example of a resolution graph, see Fig. 1. In that example, only one graph can be generated from the resolution event. From a resolution event, all resolution graphs that could be generated from the event are not always feasible, since some of them represent cases which are not physically reasonable. An example of such a case is shown in Fig. 3. Assuming independent resolution in each measurement direction (see Sec. IV), only those nodes can be connected which are nearest neighbors in at least one direction of measurement space.

The edges of a resolution graph describe the pairwise interactions between the targets. For feasible resolution graphs, we make the following assumption:

Assumption 1: The edge probabilities of a resolution graph are independent. That is, knowing that two 
targets are mutually unresolved provides no information regarding the probability that another pair of targets is unresolved.

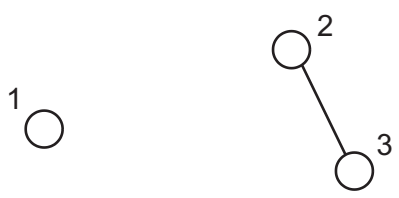

Fig. 1. Illustration of a graph, $\mathcal{G}^{1}$, which describes the resolution event that target one is resolved, while targets two and three form a group.

For the calculation of the resolution graph probability we introduce the probability, $P_{u}$, according to the following definition.

Definition 4: The probability that the pair of targets with states $\mathbf{x}_{k}^{(i)}$ and $\mathbf{x}_{k}^{(j)}$ are mutually unresolved is given by $P_{u}\left(\mathbf{x}_{k}^{(i)}, \mathbf{x}_{k}^{(j)}\right)$. Correspondingly, the probability that they are resolved is given by $1-$ $P_{u}\left(\mathbf{x}_{k}^{(i)}, \mathbf{x}_{k}^{(j)}\right)$.

The probability of the graph $\mathcal{G}^{1}$, given by Fig. 1, is thus given by

$$
\operatorname{Pr}\left\{\mathcal{G}^{1} \mid \mathbf{x}_{k}\right\}=P_{u}\left(\mathbf{x}_{k}^{(2)}, \mathbf{x}_{k}^{(3)}\right)\left(1-P_{u}\left(\mathbf{x}_{k}^{(1)}, \mathbf{x}_{k}^{(2)}\right)\right)\left(1-P_{u}\left(\mathbf{x}_{k}^{(1)}, \mathbf{x}_{k}^{(3)}\right)\right) .
$$

Example 2: Consider the resolution event $\mathcal{R}=\{\{1,2,3\}\}$ that the three targets present are all unresolved. There are exactly four graphs, $\mathcal{G}^{1}$ to $\mathcal{G}^{4}$, leading to this event, as illustrated in Fig. 2. The probability of the resolution event is then

$$
\operatorname{Pr}\left\{\mathcal{R} \mid \mathbf{x}_{k}\right\}=\operatorname{Pr}\left\{\mathcal{G}^{1} \mid \mathbf{x}_{k}\right\}+\operatorname{Pr}\left\{\mathcal{G}^{2} \mid \mathbf{x}_{k}\right\}+\operatorname{Pr}\left\{\mathcal{G}^{3} \mid \mathbf{x}_{k}\right\}+\operatorname{Pr}\left\{\mathcal{G}^{4} \mid \mathbf{x}_{k}\right\},
$$

where the respective graph probabilities are calculated similarly to the graph probability in (12).

Definition 5: A resolution graph where a node (vertex) is only connected to nodes that are the nearest neighbors in any measurement dimension is called feasible. Further, a graph which does not fulfill this is termed unfeasible.

In Fig. 2 all graphs are feasible since connections only exist to the nearest neighbors in the measurement directions (being the horizontal and vertical axes). In Fig. 3, however, the graph is unfeasible since there exists a link between nodes 1 and 4 , which are not the nearest neighbors in the $z_{1}$ dimension. That link 

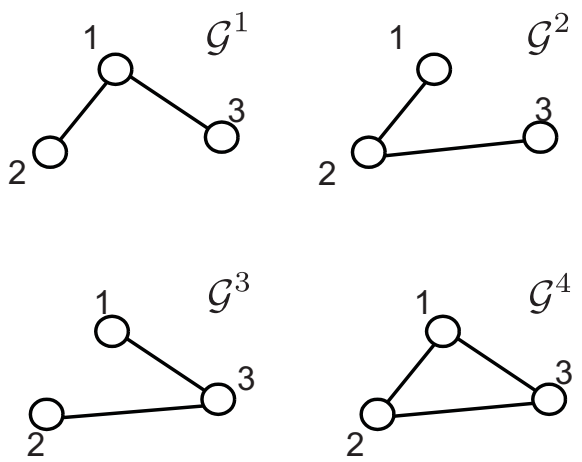

Fig. 2. Illustration of the four graphs that lead to the resolution event $\mathcal{R}$, which describes the event that a group of three targets is unresolved.

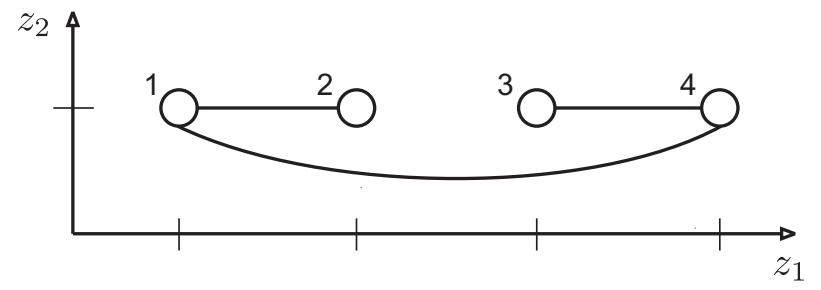

Fig. 3. Example of an unfeasible graph. In the graph, the targets 1 and 4 are connected even though they are not nearest neighbors in either the $z_{1}$ or the $z_{2}$ dimension, which makes the graph unfeasible.

thus results in the contradictory event that targets 1 and 4 are unresolved, while the more closely spaced targets 2 and 3 (in the same dimension) are resolved.

To calculate the probability for a given graph, $\mathcal{G}$, we let $\mathcal{S}_{e}$ be the set of targets that are pairwise connected by an edge, and $\mathcal{S}_{0}$ be the set of targets that are not pairwise connected. Then,

$$
\operatorname{Pr}\left\{\mathcal{G} \mid \mathbf{x}_{k}\right\}=\prod_{i=1}^{\left|\mathcal{S}_{e}\right|} P_{u}\left(\mathcal{S}_{e}(i)\right) \prod_{j=1}^{\left|\mathcal{S}_{0}\right|}\left(1-P_{u}\left(\mathcal{S}_{e}(j)\right)\right) .
$$

Note that $\mathcal{S}_{0}$ and $\mathcal{S}_{e}$ are given by $\mathcal{G}$, and $P_{u}$ is a function of the multi-target state $\mathbf{x}_{k}$. Further, we define the product over an empty set as one.

The procedure of obtaining all feasible graphs and their respective probabilities at a given time index is as follows:

1) Find all resolution events, $\mathcal{R}$.

2) For each resolution event, generate all feasible resolution graphs, $\mathcal{G}$.

3) Calculate the probability of each feasible graph using the resolution probabilities, $P_{u}$ (see (12) for an example). 


\section{B. Graph likelihood}

Apart from the probability of a graph, we would also like to describe the measurement model for a given graph $\mathcal{G}$, expressed as $p\left(\mathbf{Z}_{k} \mid \mathcal{G}, \mathbf{d}, \mathbf{x}_{k}\right)$. We call this model the graph likelihood. The data association vector $\mathbf{d}$ is included in the model since the distribution of the measurements depend on their origin. The vector is defined as

$$
\mathbf{d} \triangleq\left[\begin{array}{llll}
d_{1} & d_{2} & \ldots & d_{M_{k}}
\end{array}\right]^{T},
$$

where $d_{j}>0$ if measurement $j$ is assigned to the single target or group target $d_{j}$, and $d_{j}=0$ if the measurement is due to clutter.

Conditioned on the data association, it is known which of the measurements in $\mathbf{Z}_{k}$ are target-generated and which originate from clutter. That is, the vectors $\mathbf{Z}_{k}^{c}$ (containing $M_{k, c}$ observations) and $\mathbf{Z}_{k}^{t}$ (containing $M_{k, t}$ observations) are known, and their distributions are given by $p\left(\mathbf{Z}_{k}^{c} \mid \mathcal{G}, \mathbf{d}, \mathbf{x}_{k}\right)$ and $p\left(\mathbf{Z}_{k}^{t} \mid \mathcal{G}, \mathbf{d}, \mathbf{x}_{k}\right)$. From the assumption of spatially uniform clutter, the first density is

$$
p\left(\mathbf{Z}_{k}^{c} \mid \mathcal{G}, \mathbf{d}, \mathbf{x}_{k}\right)=\frac{1}{|\mathrm{FoV}|^{M_{k, c}}} .
$$

Further, given the data association vector $\mathbf{d}$ and the graph $\mathcal{G}$, the target-generated measurements are distributed as

$$
p\left(\mathbf{Z}_{k}^{t} \mid \mathcal{G}, \mathbf{d}, \mathbf{x}_{k}\right)=\prod_{i=1}^{M_{k, t}} p\left(\mathbf{z}_{k}^{t,(i)} \mid \mathbf{d}, \mathcal{G}, \mathbf{x}_{k}\right),
$$

where $p\left(\mathbf{z}_{k}^{t,(i)} \mid \mathbf{d}, \mathcal{G}, \mathbf{x}_{k}\right)$ is the group measurement model (see Section IV-B for one alternative). In (16), it is assumed that the measurements are independent, conditioned on their associations. Finally, the graph likelihood is given by

$$
p\left(\mathbf{Z}_{k} \mid \mathcal{G}, \mathbf{d}, \mathbf{x}_{k}\right)=\frac{1}{|\mathrm{FoV}|^{M_{k, c}}} \prod_{i=1}^{M_{k, t}} p\left(\mathbf{z}_{k}^{t,(i)} \mid \mathbf{d}, \mathcal{G}, \mathbf{x}_{k}\right),
$$

where $M_{k, c}$ and $M_{k, t}$ are known, given $\mathbf{d}$.

\section{MOdELS FOR PAIR-WISE RESOLUTION PROBABILITIES AND GROUP MEASUREMENTS}

In Section III, a novel model structure was presented for handling sensor resolution modeling for an arbitrary, but known, number of targets. The model structure relies on a graph representation of a resolution event, where each resolution graph describes the pairwise interactions between the targets, under the resolution event. 
The fundamental unit of the graph probabilities is the resolution probability $P_{u}\left(\mathbf{x}_{k}^{(i)}, \mathbf{x}_{k}^{(j)}\right)$, which states the probability that two targets with states $\mathbf{x}_{k}^{(i)}$ and $\mathbf{x}_{k}^{(j)}$ are unresolved. To complete the resolution model we hence need a model for the probability $P_{u}$. Here, we consider radar sensors, and use the two-target resolution model by Koch and van Keuk [18], presented in Section IV-A. It should be noted that the proposed resolution framework of this article is not restricted to radar sensors, and that the described radar resolution model does not rely on the use of the two-target model in [18] as a basis for the resolution probability, but that it is used due to its appealing properties.

The resolution modeling framework also includes the graph likelihood $p\left(\mathbf{Z}_{k} \mid \mathcal{G}, \mathbf{d}, \mathbf{x}_{k}\right)$. The general expression for the likelihood is given in (17). To complete that likelihood, a group measurement model is needed, which provides an explicit expression of the target measurement density $p\left(\mathbf{z}_{k}^{t,(i)} \mid \mathcal{G}, \mathbf{d}, \mathbf{x}_{k}\right)$, conditioned on a graph, an association vector and a joint target state vector. In this article, we use a simple group measurement model which is presented in Section IV-B. However, the proposed resolution model does not hinge on that group measurement model, meaning that other group measurement models are possible to use instead.

\section{A. Two-target resolution model}

For a certain pair of targets $\mathbf{x}_{k}^{(i)}, \mathbf{x}_{k}^{(j)}$ in a set $\mathcal{S}$ in (13), the probability that they are unresolved is, according to [18],

$$
P_{u}\left(\mathbf{x}_{k}^{(i)}, \mathbf{x}_{k}^{(j)}\right)=e^{-\left(\Delta \mathbf{r}^{i, j}\right)^{T}\left(\mathbf{R}_{u, N_{\mathrm{res}}}\right)^{-1} \Delta \mathbf{r}^{i, j}}
$$

where $N_{\text {res }}$ is the dimension of the measurement space ( 2 for range and azimuth), $\Delta \mathbf{r}^{i, j}$ is a vector with the distances between the predicted positions of targets $i$ and $j$ in the measurement space, and $\mathbf{R}_{u, N_{\mathrm{res}}}$ is given by

$$
\mathbf{R}_{u, N_{\mathrm{res}}}=\frac{1}{(2 \ln (2))^{N_{\mathrm{res}} / 2}} \operatorname{diag}\left\{\alpha_{1}^{2}, \ldots, \alpha_{N_{\mathrm{res}}}^{2}\right\} .
$$

The parameters $\alpha_{1}$ to $\alpha_{N_{\text {res }}}$ in (19) describe the resolution capability of the sensor in the respective measurement dimensions. Note that the diagonal structure of $\mathbf{R}_{u, N_{\mathrm{res}}}$ implies that the resolution in one dimension is independent of the resolutions in the other dimensions.

The probability $P_{u}\left(\mathbf{x}_{k}^{(i)}, \mathbf{x}_{k}^{(j)}\right)$ can also be written as a scaled multivariate Gaussian

$$
P_{u}\left(\mathbf{x}_{k}^{(i)}, \mathbf{x}_{k}^{(j)}\right)=\left|2 \pi \mathbf{R}_{u, N_{\mathrm{res}}}\right|^{1 / 2} \mathcal{N}\left(0 ; \Delta \mathbf{r}^{i, j}, \mathbf{R}_{u, N_{\text {res }}}\right) .
$$

Depending on the measurement model, the relation between $\Delta \mathbf{r}^{i, j}$ and the states $\mathbf{x}_{k}^{(i)}$ and $\mathbf{x}_{k}^{(j)}$ is either linear or non-linear. 


\section{B. Group measurement model}

For an explicit expression of the graph likelihood $p\left(\mathbf{Z}_{k} \mid \mathcal{G}, \mathbf{d}, \mathbf{x}_{k}\right)$, we need a group measurement model which describes the properties of a merged measurement from a group. The assumed model states that a group measurement can be described as a measurement of the center of gravity in the measurement dimension. That is, for a group of $n_{g}$ targets (possibly one), whose state vectors are gathered in the joint vector $\mathbf{x}_{k}^{g}$, their group measurement $\mathbf{z}_{k}^{t,(j)}$ is described by

$$
\mathbf{z}_{k}^{t,(j)}=h_{k}^{n_{g}}\left(\mathbf{x}_{k}^{g}\right)+\mathbf{u}_{k}^{g, n_{g}}
$$

where $h_{k}^{n_{g}}\left(\mathbf{x}_{k}^{g}\right)$ provides the arithmetic mean of the group targets in the measurement dimension. For linear measurement models,

$$
\mathbf{z}_{k}^{t,(j)}=\mathbf{H}_{n_{g}} \mathbf{x}_{k}^{g}+\mathbf{u}_{k}^{g, n_{g}}
$$

where

$$
\mathbf{H}_{n_{g}}=\frac{1}{n_{g}} \underbrace{[\mathbf{H}, \cdots, \mathbf{H}]}_{n_{g} \text { times }} .
$$

The vector $\mathbf{u}_{k}^{g, n_{g}} \sim \mathcal{N}\left(\mathbf{0}, \mathbf{R}_{k}^{n_{g}}\right)$ models the measurement spread from an $n_{g}$-target group, which depends on the number of targets in the group and the measurement noise. Typically, the spread increases with the number of targets $n_{g}$, due to the radar target glint phenomenon.

Other group measurement models have been presented in the literature. A model for two targets, using amplitude information, is given in [8], and is simplified in [9], while a measurement model for automotive applications is proposed in [14].

\section{CAlculation of THE POSTERIOR PROBABILITy DENSITy FUnCTION}

In this section we describe the exact calculation of the posterior probability density function (pdf) $p\left(\mathbf{x}_{k} \mid \mathbf{Z}^{k}\right)$ under unknown resolution and data association events. We start by describing the general calculation of the density, and then describe how the different parts of the general expression are obtained.

To express the posterior density, we marginalize over the feasible resolution graphs, $\mathcal{G}$, and the set of data association hypotheses $\mathcal{D}(\mathcal{G})$ for each graph. By also using Bayes' rule and the Markov property, 
we obtain

$$
\begin{aligned}
p\left(\mathbf{x}_{k} \mid \mathbf{Z}^{k}\right) & =\sum_{\mathcal{G}} \sum_{\mathbf{d} \in \mathcal{D}(\mathcal{G})} p\left(\mathbf{x}_{k}, \mathcal{G}, \mathbf{d} \mid \mathbf{Z}^{k}\right)=\sum_{\mathcal{G}} \sum_{\mathbf{d} \in \mathcal{D}(\mathcal{G})} \frac{p\left(\mathbf{Z}_{k} \mid \mathcal{G}, \mathbf{d}, \mathbf{x}_{k}\right) p\left(\mathbf{x}_{k}, \mathcal{G}, \mathbf{d} \mid \mathbf{Z}^{k-1}\right)}{p\left(\mathbf{Z}_{k} \mid \mathbf{Z}^{k-1}\right)} \\
& =\sum_{\mathcal{G}} \operatorname{Pr}\left\{\mathcal{G} \mid \mathbf{x}_{k}\right\} \sum_{\mathbf{d} \in \mathcal{D}(\mathcal{G})} \operatorname{Pr}\left\{\mathbf{d} \mid \mathcal{G}, \mathbf{x}_{k}\right\} \frac{p\left(\mathbf{Z}_{k} \mid \mathcal{G}, \mathbf{d}, \mathbf{x}_{k}\right) p\left(\mathbf{x}_{k} \mid \mathbf{Z}^{k-1}\right)}{p\left(\mathbf{Z}_{k} \mid \mathbf{Z}^{k-1}\right)}
\end{aligned}
$$

where $p\left(\mathbf{x}_{k} \mid \mathbf{Z}^{k-1}\right)$ is the predicted density of the target states, $p\left(\mathbf{Z}_{k} \mid \mathcal{G}, \mathbf{d}, \mathbf{x}_{k}\right)$ is the graph likelihood (see (17)), $\operatorname{Pr}\left\{\mathbf{d} \mid \mathcal{G}, \mathbf{x}_{k}\right\}$ is the probability of a data association hypothesis $\mathbf{d}$, and $\operatorname{Pr}\left\{\mathcal{G} \mid \mathbf{x}_{k}\right\}$ is the graph probability, given by the resolution model (see (13) for a general expression).

As seen in (25), the calculation of the posterior density includes a measurement update, given by the second sum, and a resolution model update, given by the total expression. In the following sections, these calculations are described in more detail. Note that the discussion here does not make any assumptions on the models. For calculation under Gaussian assumptions, see Section VI.

\section{A. Calculation of the predicted density}

Given the prior density $p\left(\mathbf{x}_{k-1} \mid \mathbf{Z}^{k-1}\right)$, we calculate the predicted density $p\left(\mathbf{x}_{k} \mid \mathbf{Z}^{k-1}\right)$ by means of marginalization over the previous state $\mathbf{x}_{k-1}$,

$$
\begin{aligned}
p\left(\mathbf{x}_{k} \mid \mathbf{Z}^{k-1}\right) & =\int p\left(\mathbf{x}_{k}, \mathbf{x}_{k-1} \mid \mathbf{Z}^{k-1}\right) d \mathbf{x}_{k-1}=\int p\left(\mathbf{x}_{k} \mid \mathbf{x}_{k-1}, \mathbf{Z}^{k-1}\right) p\left(\mathbf{x}_{k-1} \mid \mathbf{Z}^{k-1}\right) d \mathbf{x}_{k-1} \\
& =\int p\left(\mathbf{x}_{k} \mid \mathbf{x}_{k-1}\right) p\left(\mathbf{x}_{k-1} \mid \mathbf{Z}^{k-1}\right) d \mathbf{x}_{k-1},
\end{aligned}
$$

where we in the final step assume that the prediction process has the Markov property. In the prediction step, the prior density is propagated through the process model $p\left(\mathbf{x}_{k} \mid \mathbf{x}_{k-1}\right)$.

\section{B. Measurement update}

The measurement update includes the calculation of the data association probabilities under a resolution graph and the received data set, and the update of the predicted density function with the measurement likelihood. We start with the data association hypotheses.

1) Data association probabilities: We here state an expression for the conditional data association hypothesis probability $\operatorname{Pr}\left\{\mathbf{d} \mid \mathcal{G}, \mathbf{x}_{k}\right\}$ in (25). Using the definition of the data association vector $\mathbf{d}$ in (14),

$$
\operatorname{Pr}\left\{\mathbf{d} \mid \mathcal{G}, \mathbf{x}_{k}\right\}=P_{c}\left(M_{k, c}\right) \frac{\left(M_{k}-M_{k, t}\right) !}{M_{k} !} \prod_{\{j: \mathbf{d}(j)=0\}}\left(1-P_{D}^{j}\right) \prod_{\{j: \mathbf{d}(j)>0\}} P_{D}^{j},
$$


where $\{j: \mathbf{d}(j)=0\}$ is the set of clutter detections, $\{j: \mathbf{d}(j)>0\}$ is the set of target-generated measurements, and $P_{c}\left(M_{k, c}\right)$ is the probability of receiving $M_{k, c}$ clutter measurements, which is given by the Poisson mass function with parameter $\lambda \cdot|\mathrm{FoV}|$. Further, the detection probability $P_{D}^{j}$ for measurement index $j$ is the group detection probability, which can be modeled to attain different values for different number of targets in the group. Thus, we here assume that the detection probability only depends on the number of targets in the group, and not on their states.

2) Density update: The second part of the measurement update is to refine the predicted density $p\left(\mathbf{x}_{k} \mid \mathbf{Z}^{k-1}\right)$ with information from the current measurements, $\mathbf{Z}_{k}$, under the graph, $\mathcal{G}$ and data association $\mathbf{d} \in \mathcal{D}(\mathcal{G})$. That is, we seek an expression for the product $p\left(\mathbf{Z}_{k} \mid \mathcal{G}, \mathbf{d}, \mathbf{x}_{k}\right) p\left(\mathbf{x}_{k} \mid \mathbf{Z}^{k-1}\right)$, ignoring the scaling $p\left(\mathbf{Z}_{k} \mid \mathbf{Z}^{k-1}\right)$.

A general measurement model, under the assumption of uniformly distributed clutter in the measurement space, is given by (17). Using that model, the density product is

$$
p\left(\mathbf{Z}_{k} \mid \mathcal{G}, \mathbf{d}, \mathbf{x}_{k}\right) p\left(\mathbf{x}_{k} \mid \mathbf{Z}^{k-1}\right)=\frac{1}{|\mathrm{FoV}|^{M_{k, c}}} \prod_{i=1}^{M_{k, t}} p\left(\mathbf{z}_{k}^{t,(i)} \mid \mathcal{G}, \mathbf{d}, \mathbf{x}_{k}\right) p\left(\mathbf{x}_{k} \mid \mathbf{Z}^{k-1}\right) .
$$

The calculation of the measurement-updated pdf depends on the measurement model $p\left(\mathbf{Z}_{k} \mid \mathcal{G}, \mathbf{d}, \mathbf{x}_{k}\right)$. If it is linear and Gaussian, the first two moments of the updated density is calculated by the Kalman filter [17], for each group. For non-linear models, the Extended Kalman filter (EKF) [15] or the Unscented Kalman filter (UKF) [16] can be used. More details about explicit expressions under Gaussian assumptions are found in Section VI. The calculation also depends on the predicted density $p\left(\mathbf{x}_{k} \mid \mathbf{Z}^{k-1}\right)$.

\section{Update with the resolution model}

As seen in (25), the update with the measurement model gives an increase in the number of density components, for each graph. In the update with the resolution model, each such component is multiplied with the graph probability. For a certain graph $\mathcal{G}$ and data association hypothesis $\mathbf{d}$ we thus make the update

$$
\begin{aligned}
\operatorname{Pr}\left\{\mathcal{G} \mid \mathbf{x}_{k}\right\} & \frac{1}{|\mathrm{FoV}|^{M_{k, c}}} \prod_{i=1}^{M_{k, t}} p\left(\mathbf{z}_{k}^{t,(i)} \mid \mathcal{G}, \mathbf{d}, \mathbf{x}_{k}\right) p\left(\mathbf{x}_{k} \mid \mathbf{Z}^{k-1}\right) \\
& =\prod_{\mathcal{S}_{e}} P_{u}\left(\mathcal{S}_{e}\right) \prod_{\mathcal{S}_{0}}\left(1-P_{u}\left(\mathcal{S}_{0}\right)\right) \frac{1}{|\mathrm{FoV}|^{M_{k, c}}} \prod_{i=1}^{M_{k, t}} p\left(\mathbf{z}_{k}^{t,(i)} \mid \mathcal{G}, \mathbf{d}, \mathbf{x}_{k}\right) p\left(\mathbf{x}_{k} \mid \mathbf{z}^{k-1}\right) .
\end{aligned}
$$

Due to the $1-P_{u}$ factors, the resolution update further increases the number of density mixture components. To get a clearer insight into the update calculations needed, we study the update in more 
detail, focusing on

$$
\begin{aligned}
\prod_{i=1}^{\left|\mathcal{S}_{e}\right|} P_{u}\left(\mathcal{S}_{e}(i)\right) & \prod_{j=1}^{\left|\mathcal{S}_{0}\right|}\left(1-P_{u}\left(\mathcal{S}_{0}(j)\right)\right) \\
& =\prod_{i=1}^{\left|\mathcal{S}_{e}\right|} P_{u}\left(\mathcal{S}_{e}(i)\right)\left(1-P_{u}\left(\mathcal{S}_{0}(1)\right)\left(1-P_{u}\left(\mathcal{S}_{0}(2)\right) \times \cdots \times\left(1-P_{u}\left(\mathcal{S}_{0}\left(\left|\mathcal{S}_{0}\right|\right)\right) .\right.\right.\right.
\end{aligned}
$$

In (31), $\left|\mathcal{S}_{0}\right|$ is the cardinality of the set $\mathcal{S}_{0}$, i.e., the number of resolved pairs in the graph $\mathcal{G}$. The $\left(1-P_{u}\right)$ factors can be split up, leading to

$$
\begin{aligned}
\prod_{i=1}^{\left|\mathcal{S}_{e}\right|} P_{u}\left(\mathcal{S}_{e}(i)\right) \prod_{j=1}^{\left|\mathcal{S}_{0}\right|}\left(1-P_{u}\left(\mathcal{S}_{0}(j)\right)\right) & \left(1-\sum_{j=1}^{\left|\mathcal{S}_{0}\right|} P_{u}\left(\mathcal{S}_{0}(j)\right)+\sum_{j=1}^{\left|\mathcal{S}_{0}\right|} \sum_{\substack{l=1 \\
l \neq j}}^{\left|\mathcal{S}_{0}\right|} P_{u}\left(\mathcal{S}_{0}(j)\right) P_{u}\left(\mathcal{S}_{0}(l)\right)\right. \\
=\prod_{i=1} P_{u}\left(\mathcal{S}_{e}(i)\right) & \left.\ldots+\sum_{j=1}^{\left|\mathcal{S}_{0}\right|} \sum_{\substack{l=1 \\
l \neq j}}^{\left|\mathcal{S}_{0}\right|} \cdots \prod_{w=1}^{\left|\mathcal{S}_{0}\right|} P_{u}\left(\mathcal{S}_{0}(w)\right)\right)
\end{aligned}
$$

As seen in (18), the resolution probabilities $P_{u}\left(\mathcal{S}_{0}(j)\right)$ depend on the state vector $\mathbf{x}_{k}$. Hence, the multiplication of the measurement-updated density with a resolution probability does not only scale the density, but also affects its shape. The multiplication can thus be seen as a density update. Hence, for each term in (32), we can perform an update of the measurement-updated density. Each update can either be performed sequentially or in a single step, as described further in Section VI. The set of components generated by a resolution update, for a graph $\mathcal{G}$ and data associaiton hypothesis $\mathbf{d}$, are gathered in the set $\mathcal{U}(\mathcal{G})$.

\section{GAUSSIAN MIXTURE APPROXIMATION}

In this section, we seek to find a Gaussian-mixture expression of the posterior density $p\left(\mathbf{x}_{k} \mid \mathbf{Z}^{k}\right)$. For the sake of convenience, we assume that the prior density $p\left(\mathbf{x}_{k-1} \mid \mathbf{Z}^{k-1}\right)$ is a single Gaussian, i.e.,

$$
p\left(\mathbf{x}_{k-1} \mid \mathbf{Z}^{k-1}\right)=\mathcal{N}\left(\mathbf{x}_{k-1} ; \hat{\mathbf{x}}_{k-1 \mid k-1}, \mathbf{P}_{k-1 \mid k-1}\right) .
$$

The calculation will concern linear process and measurement models (cf. (3), (4), (21), and (22)) with Gaussian noise. The generalization to general models can be performed by linearization, similar to the EKF, or by approximations with the Unscented Transform [16]. For the probability that two targets are unresolved, $P_{u}$, we use the model in (20). 


\section{A. Prediction step}

The prediction is given by (cf. (27))

$$
p\left(\mathbf{x}_{k} \mid \mathbf{Z}^{k-1}\right)=\int p\left(\mathbf{x}_{k} \mid \mathbf{x}_{k-1}\right) p\left(\mathbf{x}_{k-1} \mid \mathbf{Z}^{k-1}\right) d \mathbf{x}_{k-1}=\mathcal{N}\left(\mathbf{x}_{k} ; \hat{\mathbf{x}}_{k \mid k-1}, \mathbf{P}_{k \mid k-1}\right),
$$

where

$$
\begin{aligned}
\hat{\mathbf{x}}_{k \mid k-1} & =\mathbf{F}_{k-1} \hat{\mathbf{x}}_{k-1 \mid k-1} \\
\mathbf{P}_{k \mid k-1} & =\mathbf{F}_{k-1} \mathbf{P}_{k-1 \mid k-1} \mathbf{F}_{k-1}^{T}+\mathbf{Q}_{k}
\end{aligned}
$$

describe the predicted mean value and covariance matrix, respectively.

\section{B. Measurement update}

In the measurement update step, the predicted density, $p\left(\mathbf{x}_{k} \mid \mathbf{Z}^{k-1}\right)$, is updated with information from the current measurement set, $\mathbf{Z}_{k}$. The update relies on the single-target and group-target measurement models. A general expression for the measurement update is given by (29). By assuming that groups are independent, the predicted density can be split into a product,

$$
p\left(\mathbf{x}_{k} \mid \mathbf{Z}^{k-1}\right)=\prod_{j=1}^{N_{g}} p\left(\mathbf{x}_{k}^{g_{j}} \mid \mathbf{Z}^{k-1}\right),
$$

where $g_{j}$ is the group index and $N_{g}$ is the number of groups. Note that the group size can be equal to one.

The measurement update step thus involves the calculation of

$$
p\left(\mathbf{Z}_{k} \mid \mathcal{G}, \mathbf{d}, \mathbf{x}_{k}\right) p\left(\mathbf{x}_{k} \mid \mathbf{Z}^{k-1}\right)=\frac{1}{|\mathrm{FoV}|^{M_{k, c}}} \prod_{i=1}^{M_{k, t}} p\left(\mathbf{z}_{k}^{t,(i)} \mid \mathcal{G}, \mathbf{d}, \mathbf{x}_{k}\right) \prod_{j=1}^{N_{g}} p\left(\mathbf{x}_{k}^{g_{j}} \mid \mathbf{Z}^{k-1}\right) .
$$

Due to the independence assumption, the update can be performed group by group. We will describe the expressions for one such update,

$$
p\left(\mathbf{z}_{k}^{t,(i)} \mid \mathcal{G}, \mathbf{d}, \mathbf{x}_{k}\right) p\left(\mathbf{x}_{k}^{g_{j}} \mid \mathbf{Z}^{k-1}\right)
$$

of group $g_{j}$ with its associated measurement $\mathbf{z}_{k}^{t,(i)}$. Using the group measurement model in (22), the update is given by

$$
p\left(\mathbf{z}_{k}^{t,(i)} \mid \mathcal{G}, \mathbf{d}, \mathbf{x}_{k}\right) p\left(\mathbf{x}_{k}^{g_{j}} \mid \mathbf{Z}^{k-1}\right)=\mathcal{N}\left(\mathbf{z}_{k}^{t,(i)} ; \mathbf{H}_{n_{g_{j}}} \mathbf{x}_{k}^{g_{j}}, \mathbf{R}_{k}^{n_{g_{j}}}\right) \mathcal{N}\left(\mathbf{x}_{k}^{g_{j}} ; \hat{\mathbf{x}}_{k \mid k-1}^{g_{j}}, \mathbf{P}_{k \mid k-1}^{g_{j}}\right) .
$$

By using the following property of a Gaussian product

$$
\mathcal{N}(\mathbf{x} ; \hat{\mathbf{x}}, \mathbf{P}) \mathcal{N}(\mathbf{z} ; \mathbf{H x}, \mathbf{R})=\mathcal{N}(\mathbf{x} ; \boldsymbol{\mu}, \mathbf{\Sigma}) \mathcal{N}(\mathbf{z} ; \hat{\mathbf{z}}, \mathbf{S})
$$


where

$$
\begin{aligned}
\hat{\mathbf{z}} & =\mathbf{H} \hat{\mathbf{x}} \\
\mathbf{S} & =\mathbf{H} \mathbf{P} \mathbf{H}^{T}+\mathbf{R} \\
\boldsymbol{\mu} & =\hat{\mathbf{x}}+\mathbf{K}(\mathbf{z}-\mathbf{H} \hat{\mathbf{x}}) \\
\mathbf{\Sigma} & =(\mathbf{I}-\mathbf{K} \mathbf{H}) \mathbf{P} \\
\mathbf{K} & =\mathbf{P} \mathbf{H}^{T} \mathbf{S}^{-1},
\end{aligned}
$$

we obtain

$$
\mathcal{N}\left(\mathbf{z}_{k}^{t,(i)} ; \mathbf{H}_{n_{g_{j}}} \mathbf{x}_{k}^{g_{j}}, \mathbf{R}_{k}^{n_{g_{j}}}\right) \mathcal{N}\left(\mathbf{x}_{k}^{g_{j}} ; \hat{\mathbf{x}}_{k \mid k-1}^{g_{j}}, \mathbf{P}_{k \mid k-1}^{g_{j}}\right)=\mathcal{N}\left(\mathbf{z}_{k}^{t,(i)} ; \hat{\mathbf{z}}_{k}^{g_{j}}, \mathbf{S}^{g_{j}}\right) \mathcal{N}\left(\mathbf{x}_{k}^{g_{j}} ; \hat{\mathbf{x}}_{k \mid k}^{g_{j}}, \mathbf{P}_{k \mid k}^{g_{j}}\right)
$$

where the mean values and covariance matrices are given by identification from (40)-(45).

The update can also be performed in a single step. For that we utilize the multi-target measurement model in (8). Thereby,

$$
\begin{aligned}
p\left(\mathbf{Z}_{k} \mid \mathcal{G}, \mathbf{d}, \mathbf{x}_{k}\right) p\left(\mathbf{x}_{k} \mid \mathbf{Z}^{k-1}\right) & =\mathcal{N}\left(\mathbf{Z}_{k} ; \breve{\mathbf{H}} \mathbf{x}_{k}, \mathbf{R} \otimes \mathbf{I}_{M_{k, t} \times M_{k, t}}\right) \mathcal{N}\left(\mathbf{x}_{k} ; \hat{\mathbf{x}}_{k \mid k-1}, \mathbf{P}_{k \mid k-1}\right) \\
& =\mathcal{N}\left(\mathbf{Z}_{k} ; \breve{\mathbf{H}} \hat{\mathbf{x}}_{k \mid k-1}, \mathbf{S}^{\mathcal{G}, \mathbf{d}}\right) \mathcal{N}\left(\mathbf{x}_{k} ; \hat{\mathbf{x}}_{k \mid k}, \mathbf{P}_{k \mid k}\right)
\end{aligned}
$$

where

$$
\breve{\mathbf{H}}=\mathbf{C}_{k}^{\mathcal{G}, \mathbf{d}} \otimes \mathbf{I}_{N_{z} \times N_{z}} \tilde{\mathbf{H}}
$$

describes the relation between target states and the joint measurement vector, in which $\mathbf{C}_{k}^{\mathcal{G}, \mathbf{d}}$ represents the current resolution and data association events. By identification from (40)-(45),

$$
\begin{aligned}
\hat{\mathbf{Z}}_{k}^{\mathcal{G}, \mathbf{d}} & =\breve{\mathbf{H}} \hat{\mathbf{x}}_{k \mid k-1} \\
\mathbf{S}^{\mathcal{G}, \mathbf{d}} & =\breve{\mathbf{H}} \mathbf{P}_{k \mid k-1} \breve{\mathbf{H}}^{T} \\
\mathbf{K}^{\mathcal{G}, \mathbf{d}} & =\mathbf{P}_{k \mid k-1} \breve{\mathbf{H}}^{T}\left(\mathbf{S}^{\mathcal{G}, \mathbf{d}}\right)^{-1} \\
\hat{\mathbf{x}}_{k \mid k} & =\hat{\mathbf{x}}_{k \mid k-1}+\mathbf{K}^{\mathcal{G}, \mathbf{d}}\left(\mathbf{Z}_{k}-\breve{\mathbf{H}}_{k \mid k-1}\right) .
\end{aligned}
$$

\section{Sequential update with the resolution model}

As seen in (30) and (32), the resolution model update involves the calculation of the product between a measurement-updated density and a sum of products. The update can be performed summand by summand, producing a density mixture. For each summand, the updated density is multiplied with a 
product of $P_{u}$ factors. This calculation can either be carried out sequentially, factor by factor, or in a single step. Here we describe the sequential update.

Let us start with a single $P_{u}$ factor, $P_{u}\left(\mathbf{x}_{k}^{(i)}, \mathbf{x}_{k}^{(j)}\right)$. From (48), the updated density is described by a product of two Gaussian densities, where only one depends on $\mathbf{x}_{k}$. The resolution model update is thus

$$
P_{u}\left(\mathbf{x}_{k}^{(i)}, \mathbf{x}_{k}^{(j)}\right) \mathcal{N}\left(\mathbf{x}_{k} ; \hat{\mathbf{x}}_{k \mid k}, \mathbf{P}_{k \mid k}\right)=\left|2 \pi \mathbf{R}_{u, N_{\text {res }}}\right|^{1 / 2} \mathcal{N}\left(0 ; \Delta \mathbf{r}^{i, j}, \mathbf{R}_{u, N_{\text {res }}}\right) \mathcal{N}\left(\mathbf{x}_{k} ; \hat{\mathbf{x}}_{k \mid k}, \mathbf{P}_{k \mid k}\right),
$$

where

$$
\Delta \mathbf{r}^{i, j}=\mathbf{H}\left(\mathbf{x}_{k}^{(i)}-\mathbf{x}_{k}^{(j)}\right)
$$

To describe the product of Gaussians, we would like to express the resolution model Gaussian as a function of the joint target state vector $\mathbf{X}_{k}$. To do so, we use the Kronecker delta

$$
\delta_{k, i} \triangleq \begin{cases}1 & \text { if } k=i \\ 0 & \text { otherwise }\end{cases}
$$

the $1 \times N$ vector

$$
\pi^{(i, j)} \triangleq\left[\delta_{1, i}-\delta_{1, j}, \ldots, \delta_{N, i}-\delta_{N, j}\right]
$$

and the $N_{z} \times\left(N \cdot N_{z}\right)$ matrix

$$
\boldsymbol{\Pi}^{(i, j)}=\pi^{(i, j)} \otimes \mathbf{I}_{N_{z}}
$$

Then,

$$
\Delta \mathbf{r}^{i, j}=\boldsymbol{\Pi}^{(i, j)} \tilde{\mathbf{H}} \mathbf{x}_{k}
$$

where $\tilde{\mathbf{H}}$ is defined in (9).

The update with one $P_{u}$ factor is hence

$$
P_{u}\left(\mathbf{x}_{k}^{(i)}, \mathbf{x}_{k}^{(j)}\right) \mathcal{N}\left(\mathbf{x}_{k} ; \hat{\mathbf{x}}_{k \mid k}, \mathbf{P}_{k \mid k}\right)=\left|2 \pi \mathbf{R}_{u, N_{\mathrm{res}}}\right|^{1 / 2} \mathcal{N}\left(\mathbf{0} ; \mathbf{\Pi}^{(i, j)} \tilde{\mathbf{H}} \mathbf{x}_{k}, \mathbf{R}_{u, N_{\mathrm{res}}}\right) \mathcal{N}\left(\mathbf{x}_{k} ; \hat{\mathbf{x}}_{k \mid k}, \mathbf{P}_{k \mid k}\right)
$$

when the measurement model is linear. This can be re-written as

$$
\mathcal{N}\left(\mathbf{0} ; \boldsymbol{\Pi}^{(i, j)} \tilde{\mathbf{H}} \mathbf{x}_{k}, \mathbf{R}_{u, N_{\mathrm{res}}}\right) \mathcal{N}\left(\mathbf{x}_{k} ; \hat{\mathbf{x}}_{k \mid k}, \mathbf{P}_{k \mid k}\right)=\mathcal{N}\left(\mathbf{0} ; \mathbf{\Pi}^{(i, j)} \tilde{\mathbf{H}} \hat{\mathbf{x}}_{k \mid k}, \mathbf{S}^{i, j}\right) \mathcal{N}\left(\mathbf{x}_{k} ; \hat{\mathbf{x}}_{k \mid k}^{i, j}, \mathbf{P}_{k \mid k}^{i, j}\right)
$$

where $\hat{\mathbf{x}}_{k \mid k}^{i, j}$ and $\mathbf{P}_{k \mid k}^{i, j}$ describe the expected value and the covariance matrix of the target states given measurement update under hypothesis $\mathbf{d}$, for graph $\mathcal{G}$, and resolution model update for the unresolved target pair $(i, j)$. The exact form of $\mathbf{S}^{i, j}, \hat{\mathbf{x}}_{k \mid k}^{i, j}$ and $\mathbf{P}_{k \mid k}^{i, j}$ are given by identification from (40)-(45). As seen 
in the update equation, the 'negative information' from a missed detection due to resolution limitations is hence incorporated in the tracking framework as a measured ' 0 ' of the separation of the target pair.

When the summands in (32) involves several $P_{u}$ factors, similar calculations as in (60) are performed sequentially, factor by factor. That is, if we have a second factor $P_{u}\left(\mathbf{x}_{k}^{(l)}, \mathbf{x}_{k}^{(m)}\right)$, we perform a similar calculation of

$$
P_{u}\left(\mathbf{x}_{k}^{(l)}, \mathbf{x}_{k}^{(m)}\right) \mathcal{N}\left(\mathbf{x}_{k} ; \hat{\mathbf{x}}_{k \mid k}^{i, j}, \mathbf{P}_{k \mid k}^{i, j}\right)
$$

and so on. In general (cf. (32)),

$$
\begin{aligned}
& \prod_{w=1}^{\left|\mathcal{S}_{0}\right|} P_{u}\left(\mathcal{S}_{0}(w) \mathcal{N}\left(\mathbf{x}_{k} ; \hat{\mathbf{x}}_{k \mid k}, \mathbf{P}_{k \mid k}\right)\right. \\
& \quad=\left|2 \pi \mathbf{R}_{u, N_{\text {res }}}\right| \prod_{w=1}^{\left|\mathcal{S}_{0}\right| / 2} \prod^{\left|\mathcal{S}_{0}\right|} \mathcal{N}\left(\mathbf{0} ; \mathbf{\Pi}^{(w)} \tilde{\mathbf{H}} \hat{\mathbf{x}}_{k \mid k}^{w-1}, \mathbf{S}_{k \mid k}^{w}\right) \mathcal{N}\left(\mathbf{x}_{k} ; \hat{\mathbf{x}}_{k \mid k}^{\mathcal{S}_{0}}, \mathbf{P}_{k \mid k}^{\mathcal{S}_{0}}\right),
\end{aligned}
$$

where $\hat{\mathbf{x}}^{w-1}$ is the state estimate after the update with resolution pair $w-1$. Further, $\mathbf{S}^{w}$ depends on the previous covariance $\mathbf{P}_{k \mid k}^{w-1}$ (cf. (42)), where we define $\hat{\mathbf{x}}_{k \mid k}^{0} \triangleq \hat{\mathbf{x}}_{k \mid k}$, and where $\hat{\mathbf{x}}_{k \mid k}^{\mathcal{S}_{0}}$ and $\mathbf{P}_{k \mid k}^{\mathcal{S}_{0}}$ are the state estimate and covariance matrix after the sequence of updates, for the set $\mathcal{S}_{0}$.

\section{One-step update with the resolution model}

Instead of making a sequential resolution update, as in the previous section, we can make a single update for each summand in (32). To do so, we first note that the product of $P_{u}$ factors (cf. (32)) can be written as

$$
\begin{aligned}
\prod_{w=1}^{\left|\mathcal{S}_{0}\right|} P_{u}\left(\mathcal{S}_{0}(w)\right) & =e^{-\left(\Delta \mathbf{r}^{i, j}\right)^{T}\left(\mathbf{R}_{u, N_{\mathrm{res}}}\right)^{-1} \Delta \mathbf{r}^{i, j}}=\prod_{w=1}^{\left|\mathcal{S}_{0}\right|} e^{-\left(\boldsymbol{\Pi}^{(i, j)} \tilde{\mathbf{H}} \mathbf{x}_{k}\right)^{T}\left(\mathbf{R}_{u, N_{\mathrm{res}}}\right)^{-1} \Pi^{(i, j)} \tilde{\mathbf{H}} \mathbf{x}_{k}} \\
& =e^{-\frac{1}{2} \sum_{w=1}^{\left|\mathcal{S}_{0}\right|}\left(\boldsymbol{\Pi}^{(i, j)} \tilde{\mathbf{H}} \mathbf{x}_{k}\right)^{T}\left(\mathbf{R}_{u, N_{\mathrm{res}}}\right)^{-1} \Pi^{(i, j)} \tilde{\mathbf{H}} \mathbf{x}_{k}} \\
& =e^{-\frac{1}{2} \mathbf{x}_{k}^{T}\left\{\sum_{w=1}^{\left|\mathcal{S}_{0}\right|}\left(\Pi^{(i, j)} \tilde{\mathbf{H}}\right)^{T}\left(\mathbf{R}_{u, N_{\mathrm{res}}}\right)^{-1} \Pi^{(i, j)} \tilde{\mathbf{H}}\right\} \mathbf{x}_{k}}=e^{-\frac{1}{2} \mathbf{x}_{k}^{T} \tilde{\mathbf{R}}_{u}^{-1} \mathbf{x}_{k}} .
\end{aligned}
$$

By using the Kronecker product,

$$
\left.\tilde{\mathbf{R}}_{u}^{-1} \triangleq \sum_{w=1}^{\left|\mathcal{S}_{0}\right|}\left(\boldsymbol{\Pi}^{(i, j)} \tilde{\mathbf{H}}\right)^{T}\left(\mathbf{R}_{u, N_{\text {res }}}\right)^{-1} \boldsymbol{\Pi}^{(i, j)} \tilde{\mathbf{H}}=\sum_{w=1}^{\left|\mathcal{S}_{0}\right|} \tilde{\mathbf{H}}^{T}\left\{\left(\pi^{(i, j)}\right)^{T} \pi^{(i, j)}\right) \otimes \mathbf{R}_{u, N_{\text {res }}}^{-1}\right\} \tilde{\mathbf{H}} .
$$

The matrices $\tilde{\mathbf{H}}$ and $\mathbf{R}_{u, N_{\text {res }}}$ do not depend on $i$ and $j$ and can hence be moved out of the summation. So, by defining

$$
\left.\mathbf{G} \triangleq \sum_{w=1}^{\left|\mathcal{S}_{0}\right|}\left(\pi^{(i, j)}\right)^{T} \pi^{(i, j)}\right)
$$


we get

$$
\tilde{\mathbf{R}}_{u}^{-1}=\tilde{\mathbf{H}}^{T}\left(\mathbf{G} \otimes \mathbf{R}_{u, N_{\mathrm{res}}}^{-1}\right) \tilde{\mathbf{H}}
$$

Since $\mathbf{G}$ is positive semi-definite, it has a matrix square root, $\mathbf{D}$, and we can thus write

$$
\mathbf{G}=\mathbf{D}^{T} \mathbf{D}
$$

Then, repeatedly using the Kronecker product rule [7]

$$
(\mathbf{A} \otimes \mathbf{B})(\mathbf{C} \otimes \mathbf{D})=(\mathbf{A C}) \otimes(\mathbf{B D})
$$

we have

$$
\begin{aligned}
\mathbf{G} \otimes \mathbf{R}_{u, N_{\mathrm{res}}}^{-1} & =\left(\mathbf{D}^{T} \mathbf{D}\right) \otimes \mathbf{R}_{u, N_{\mathrm{res}}}^{-1}=\left(\mathbf{D}^{T} \otimes \mathbf{R}_{u, N_{\mathrm{res}}}^{-1}\right)\left(\mathbf{D} \otimes \mathbf{I}_{N_{\mathrm{resr}}}\right) \\
& =\left(\mathbf{D}^{T} \otimes \mathbf{I}_{N_{\mathrm{res}}}\right)\left(\mathbf{I}_{N} \otimes \mathbf{R}_{u, N_{\mathrm{res}}}^{-1}\right)\left(\mathbf{D} \otimes \mathbf{I}_{N_{\mathrm{res}}}\right) .
\end{aligned}
$$

Since

$$
\mathbf{D}^{T} \otimes \mathbf{I}_{N_{\mathrm{res}}}=\mathbf{D}^{T} \otimes \mathbf{I}_{N_{\mathrm{res}}}^{T}=\left(\mathbf{D} \otimes \mathbf{I}_{N_{\mathrm{res}}}\right)^{T}
$$

the matrix $\tilde{\mathbf{R}}_{u}^{-1}$ can be written as

$$
\tilde{\mathbf{R}}_{u}^{-1}=\tilde{\mathbf{H}}^{T}\left(\mathbf{D} \otimes \mathbf{I}_{N_{\mathrm{res}}}\right)^{T}\left(\mathbf{I}_{N} \otimes \mathbf{R}_{u, N_{\mathrm{res}}}^{-1}\right)\left(\mathbf{D} \otimes \mathbf{I}_{N_{\mathrm{res}}}\right) \tilde{\mathbf{H}} .
$$

So, through

$$
\begin{aligned}
\mathbf{x}_{k}^{T} \tilde{\mathbf{R}}_{u}^{-1} \mathbf{x}_{k} & =\mathbf{x}_{k}^{T} \tilde{\mathbf{H}}^{T}\left(\mathbf{D} \otimes \mathbf{I}_{N_{\mathrm{res}}}\right)^{T} \times\left(\mathbf{I}_{N} \otimes \mathbf{R}_{u, N_{\mathrm{res}}}^{-1}\right)\left(\mathbf{D} \otimes \mathbf{I}_{N_{\mathrm{res}}}\right) \tilde{\mathbf{H}} \mathbf{x}_{k} \\
& =\left(\left(\mathbf{D} \otimes \mathbf{I}_{N_{\mathrm{res}}}\right) \tilde{\mathbf{H}} \mathbf{x}_{k}\right)^{T}\left(\mathbf{I}_{N} \otimes \mathbf{R}_{u, N_{\mathrm{res}}}^{-1}\right)\left(\left(\mathbf{D} \otimes \mathbf{I}_{N_{\mathrm{res}}}\right) \tilde{\mathbf{H}} \mathbf{x}\right),
\end{aligned}
$$

the probability in (65) is described. By further using that

$$
\left(\mathbf{I}_{N} \otimes \mathbf{R}_{u, N_{\mathrm{res}}}^{-1}\right)=\left(\mathbf{I}_{N} \otimes \mathbf{R}_{u, N_{\mathrm{res}}}\right)^{-1}
$$

the product of $P_{u}$ factors can be written as a scaled Gaussian density

$$
\prod_{w=1}^{\left|\mathcal{S}_{0}\right|} P_{u}\left(\mathcal{S}_{0}(w)=\left|2 \pi \mathbf{I}_{N} \otimes \mathbf{R}_{u, N_{\mathrm{res}}}\right| \mathcal{N}\left(\mathbf{0} ;\left(\mathbf{D} \otimes \mathbf{I}_{N_{\mathrm{res}}}\right) \tilde{\mathbf{H}} \mathbf{x}_{k}, \mathbf{I}_{N} \otimes \mathbf{R}_{u, N_{\mathrm{res}}}\right) .\right.
$$

Using the description in (78), the update of the measurement-updated density with one of the summation terms in (32) can be performed in a single Gaussian-product step (the updated density, is for example given by (48))

$$
\begin{aligned}
\mathcal{N}\left(\mathbf{0} ;\left(\mathbf{D} \otimes \mathbf{I}_{N_{\mathrm{res}}}\right)\right. & \left.\tilde{\mathbf{H}} \mathbf{x}_{k}, \mathbf{I}_{N} \otimes \mathbf{R}_{u, N_{\mathrm{res}}}\right) \mathcal{N}\left(\mathbf{x}_{k} ; \hat{\mathbf{x}}_{k \mid k}, \mathbf{P}_{k \mid k}\right) \\
& =\mathcal{N}\left(\mathbf{0} ;\left(\mathbf{D} \otimes \mathbf{I}_{N_{\mathrm{res}}}\right) \tilde{\mathbf{H}} \hat{\mathbf{x}}_{k \mid k}, \mathbf{S}^{u}\right) \mathcal{N}\left(\mathbf{x}_{k} ; \hat{\mathbf{x}}_{k \mid k}^{u}, \mathbf{P}_{k \mid k}^{u}\right)
\end{aligned}
$$


where

$$
\begin{aligned}
\mathbf{S}^{u} & =\left(\left(\mathbf{D} \otimes \mathbf{I}_{N_{\mathrm{res}}}\right) \tilde{\mathbf{H}}\right)^{T} \mathbf{P}_{k \mid k}\left(\left(\mathbf{D} \otimes \mathbf{I}_{N_{\mathrm{res}}}\right) \tilde{\mathbf{H}}\right)+\mathbf{I}_{N} \otimes \mathbf{R}_{u, N_{\mathrm{res}}} \\
\mathbf{K}^{u} & =\mathbf{P}_{k \mid k}\left(\left(\mathbf{D} \otimes \mathbf{I}_{N_{\mathrm{res}}}\right) \tilde{\mathbf{H}}\right)^{T}\left(\mathbf{S}^{u}\right)^{-1} \\
\hat{\mathbf{x}}_{k \mid k} & =\mathbf{x}_{k \mid k}+\mathbf{K}^{u}\left(\mathbf{0}-\left(\mathbf{D} \otimes \mathbf{I}_{N_{\mathrm{res}}}\right) \tilde{\mathbf{H}} \hat{\mathbf{x}}_{k \mid k}\right) \\
\mathbf{P}_{k \mid k}^{u} & =\left(\mathbf{I}-\mathbf{K}^{u}\left(\mathbf{D} \otimes \mathbf{I}_{N_{\mathrm{res}}}\right) \tilde{\mathbf{H}}\right) \mathbf{P}_{k \mid k} .
\end{aligned}
$$

The one-step update described here is performed for each term in the sums over $\left|\mathcal{S}_{0}\right|$ in (32). The resolution model update is then finalized by the update with the product of $P_{u}\left(\mathcal{S}_{e}\right)$ factors, which can also be performed in a single calculation for each term.

\section{E. Summary}

The update with measurement and resolution models can be performed in four different ways:

1) Sequential measurement and resolution updates.

2) Sequential measurement update and one-step resolution update.

3) One-step measurement update and sequential resolution update.

4) One-step measurement and resolution updates.

The advantage with the one-step updates is that they keep the form of the Gaussian mixture throughout the updates, since no assumption about independence between groups is necessary. The drawback, however, is that the calculations require high-dimensional matrix operations. Then, the sequential update is a further approximation which makes it faster but leads to the effect that the order matters and that graph feasibility might not be preserved.

\section{Joint Probabilistic DAta Association FILTERING USING THE RESOLUTION MOdel}

In Section VI, the calculation of the posterior density was described under Gaussian assumptions. If the prior density is Gaussian, the posterior density is a Gaussian mixture, where the number of components depend on

- the number of resolution graphs, $\mathcal{G}$,

- the number of open links in the graphs, yielding $\left(1-P_{u}\right)$ factors which doubles the number of components, and

- the number of data association hypotheses $|\mathcal{D}(\mathcal{G})|$ for each graph. 
Since processing of the full Gaussian mixture is infeasible, due to memory and processing limitations, approximations are necessary. For the data association problem, a common algorithm is the Joint Probabilistic Data Association (JPDA) filter [13]. The approach of the filter is to, at each time step, approximate a Gaussian mixture with a single Gaussian, using moment matching. In [5], the JPDA algorithm was extended with a resolution model for the two-target case. Here, we describe extensions of the JPDA algorithm under resolution limitations for arbitrary target numbers. For the extension, two alternatives are proposed: either to calculate the full Gaussian mixture, and to approximate that with a single Gaussian, or to perform a two-step approximation, which is less computationally intensive. The two approaches are described in the following two sections.

\section{A. JPDA approximation of the full Gaussian mixture}

The most accurate moment-matching approximation is given by calculating the full Gaussian mixture in (87), and then approximate that with a single Gaussian. A pseudo-code description of such a procedure is found in Table I, and in the following we discuss the different steps in more detail. The algorithmic description starts with the predicted density $p\left(\mathbf{x}_{k} \mid \mathbf{Z}^{k-1}\right)$.

Step I: Generate all feasible graphs: The first step is to generate all feasible graphs, $\mathcal{G}$, which is done by first forming the set of possible resolution events, $\mathcal{R}$ (cf., for example, Example 1), and then generating the feasible resolution graphs for each event.

Step II: Data association hypotheses formulation: For each generated resolution graph, the set of data association hypotheses, $\mathcal{D}(\mathcal{G})$ is formulated. The set of data association hypotheses includes all combinations of group target-to-measurement assignments ${ }^{3}$, including missed detections.

Step III: Measurement and resolution model update: The third step is divided into three sub-steps. All operations in step III are performed for each data association hypothesis formulated in Step II.

In Step III-a), the data association hypothesis probability is calculated according to (28).

Step III-b) is to update the predicted density function $\mathcal{N}\left(\mathbf{x}_{k} ; \hat{\mathbf{x}}_{k \mid k}, \mathbf{P}_{k \mid k}\right)$ with the measurement likelihood under the current resolution and data association hypotheses. The update can either be performed sequentially, as described in (38) and (39), or in a single calculation, given by (48). After the measurement update, we obtain the scaled Gaussian density $c^{\mathcal{G}, \mathbf{d}} \mathcal{N}\left(\mathbf{x}_{k} ; \hat{\mathbf{x}}_{k \mid k}^{\mathcal{G}, \mathbf{d}}, \mathbf{P}_{k \mid k}^{\mathcal{G}, \mathbf{d}}\right)$ with proportionality

\footnotetext{
${ }^{3}$ Note that several graphs lead to the same data association hypothesis. Thus, in the measurement update, only a single update is needed for those graphs. The resolution update with the graph probabilities, however, must be performed for each graph.
} 
TABLE I

COMPLETE GAUSSIAN MIXTURE JPDA FILTER WITH RESOLUTION MODEL

I: Generate all feasible graphs, $\mathcal{G}$.

II : For each graph, formulate all data association hypotheses, $\mathcal{D}(\mathcal{G})$.

III: For each data association hypothesis, $\mathbf{d} \in \mathcal{D}(\mathcal{G})$ :

III-a) Calculate $\operatorname{Pr}\left\{\mathbf{d} \mid \mathcal{G}, \mathbf{x}_{k}\right\}$ according to (28).

III-b) Perform measurement update according to (38) and (39), or by (48). This yields a scaled Gaussian $c^{\mathcal{G}, \mathbf{d}} \mathcal{N}\left(\mathbf{x}_{k} ; \hat{\mathbf{x}}_{k \mid k}^{\mathcal{G}, \mathbf{d}}, \mathbf{P}_{k \mid k}^{\mathcal{G}, \mathbf{d}}\right)$ with weight given by (84).

III-c) Update with resolution model according to (30), (32), and either (60)-(62), or by (78) and (79). The result is a sum of scaled Gaussians $\sum_{u \in \mathcal{U}(\mathcal{G})} c^{\mathcal{G}, u, \mathbf{d}} \mathcal{N}\left(\mathbf{x}_{k} ; \hat{\mathbf{x}}_{k \mid k}^{\mathcal{G}, u, \mathbf{d}}, \mathbf{P}^{\mathcal{G}, u, \mathbf{d}}\right)$ with weights given by $(85)$.

IV: Approximate the Gaussian mixture in (87) using moment matching, according to (86), and (88)-(90).

weight (using (48))

$$
c^{\mathcal{G}, \mathbf{d}}=\operatorname{Pr}\left\{\mathbf{d} \mid \mathcal{G}, \mathbf{x}_{k}\right\} \mathcal{N}\left(\mathbf{Z}_{k} ; \breve{\mathbf{H}} \hat{\mathbf{x}}_{k \mid k-1}, \mathbf{S}^{\mathcal{G}, \mathbf{d}}\right) .
$$

In Step III-c), the resolution update is performed for each data association hypothesis. The general update is described in (30). As seen in (32), the resolution update results in a sum of products. For each summation term, the calculation can either be performed sequentially, as given by (60), (61) and (62), or in a single step, described by (78) and (79). After the update, we obtain a sum of scaled Gaussians $\sum_{u \in \mathcal{U}(\mathcal{G})} c^{\mathcal{G}, u, \mathbf{d}} \mathcal{N}\left(\mathbf{x}_{k} ; \hat{\mathbf{x}}_{k \mid k}^{\mathcal{G}, u, \mathbf{d}}, \mathbf{P}^{\mathcal{G}, u, \mathbf{d}}\right)$, where the weight is given by

$$
c^{\mathcal{G}, u, \mathbf{d}}=\operatorname{Pr}\left\{\mathbf{d} \mid \mathcal{G}, \mathbf{x}_{k}\right\} \mathcal{N}\left(\mathbf{Z}_{k} ; \breve{\mathbf{H}} \hat{\mathbf{x}}_{k \mid k-1}, \mathbf{S}^{\mathcal{G}, \mathbf{d}}\right)\left|2 \pi \mathbf{I}_{N} \otimes \mathbf{R}_{u, N_{\text {res }}}\right| \mathcal{N}\left(\mathbf{0} ;\left(\mathbf{D}^{u} \otimes \mathbf{I}_{N_{\text {res }}}\right) \tilde{\mathbf{H}} \hat{\mathbf{x}}_{k \mid k}, \mathbf{S}^{u}\right) .
$$

In (85), $\mathbf{D}^{u}$ represent the current resolution event (cf. (67) and (69)).

Step IV: Moment matching: The final step of the full Gaussian mixture JPDA algorithm with resolution modeling is the moment matching approximation. First, the weight components are normalized,

$$
\bar{c}^{\mathcal{G}, u, \mathbf{d}}=\frac{c^{\mathcal{G}, u, \mathbf{d}}}{\sum_{\mathcal{G}} \sum_{u \in \mathcal{U}(\mathcal{G})} \sum_{\mathbf{d} \in \mathcal{D}(\mathcal{G})} c^{\mathcal{G}, u, \mathbf{d}}} .
$$

If the prior density is Gaussian, the end result after resolution and measurement updates is a density mixture of the form

$$
p\left(\mathbf{x}_{k} \mid \mathbf{Z}^{k}\right)=\sum_{\mathcal{G}} \sum_{u \in \mathcal{U}(\mathcal{G})} \sum_{\mathbf{d} \in \mathcal{D}(\mathcal{G})} \bar{c}^{\mathcal{G}, u, \mathbf{d}} \mathcal{N}\left(\mathbf{x}_{k} ; \hat{\mathbf{x}}_{k \mid k}^{\mathcal{G}, u, \mathbf{d}}, \mathbf{P}_{k \mid k}^{\mathcal{G}, u, \mathbf{d}}\right)
$$


The final step of the first version of JPDA algorithm is to approximate this Gaussian mixture density with a single Gaussian. The best way of making that approximation, in the Kullback-Leibler sense, is to perform second-order moment matching. We thus make the approximation

$$
p\left(\mathbf{x}_{k} \mid \mathbf{Z}^{k}\right) \approx \mathcal{N}\left(\mathbf{x}_{k} ; \hat{\mathbf{x}}_{k \mid k}^{A_{1}}, \mathbf{P}_{k \mid k}^{A_{1}}\right)
$$

where

$$
\begin{aligned}
\hat{\mathbf{x}}_{k \mid k}^{A_{1}} & =\sum_{\mathcal{G}} \sum_{u \in \mathcal{U}(\mathcal{G})} \sum_{\mathbf{d} \in \mathcal{D}(\mathcal{G})} \bar{c}^{\mathcal{G}, u, \mathbf{d}} \hat{\mathbf{x}}_{k \mid k}^{\mathcal{G}, u, \mathbf{d}} \\
\mathbf{P}_{k \mid k}^{A_{1}} & =\sum_{\mathcal{G}} \sum_{u \in \mathcal{U}(\mathcal{G})} \sum_{\mathbf{d} \in \mathcal{D}(\mathcal{G})} \bar{c}^{\mathcal{G}, u, \mathbf{d}}\left\{\mathbf{P}_{k \mid k}^{\mathcal{G}, u, \mathbf{d}}+\left(\hat{\mathbf{x}}_{k \mid k}^{\mathcal{G}, u, \mathbf{d}}-\hat{\mathbf{x}}_{k \mid k}^{A_{1}}\right)\left(\hat{\mathbf{x}}_{k \mid k}^{\mathcal{G}, u, \mathbf{d}}-\hat{\mathbf{x}}_{k \mid k}^{A_{1}}\right)^{T}\right\} .
\end{aligned}
$$

\section{B. Two-step JPDA approximation}

When the number of targets is large and the clutter level high, the number of Gaussian components to calculate in each iteration can be large. Then, a less computationally demanding method is advantageous. We here present such a method, where instead of approximating the full Gaussian mixture, we perform a two-step procedure including two Gaussian approximations.

In Table II, the two-step JPDA filter is described. In the following, we describe some of the steps in more detail. Since Step I to III-b) are the same as for the algorithm in Table I, we start with Step IV.

Step IV: Moment matching over data association hypotheses: After the measurement update step for graph $\mathcal{G}$ and data association hypothesis $\mathbf{d}$, we obtain a scaled Gaussian $c^{\mathcal{G}, \mathbf{d}} \mathcal{N}\left(\mathbf{x}_{k} ; \hat{\mathbf{x}}_{k \mid k}^{\mathcal{G}, \mathbf{d}}, \mathbf{P}_{k \mid k}^{\mathcal{G}, \mathbf{d}}\right)$ with weight given by (84). After having performed measurement updates for all data association hypotheses, we have a Gaussian mixture. Step IV of the two-step JPDA filter is to approximate this Gaussian mixture with a single Gaussian, using moment matching ${ }^{4}$, i.e.,

$$
\sum_{\mathbf{d} \in \mathcal{D}(\mathcal{G})} \bar{c}^{\mathcal{G}, \mathbf{d}} \mathcal{N}\left(\mathbf{x}_{k} ; \hat{\mathbf{x}}_{k \mid k}^{\mathcal{G}, \mathbf{d}}, \mathbf{P}_{k \mid k}^{\mathcal{G}, \mathbf{d}}\right) \approx \mathcal{N}\left(\mathbf{x}_{k} ; \hat{\mathbf{x}}_{k \mid k}^{\mathcal{G}}, \mathbf{P}_{k \mid k}^{\mathcal{G}}\right)
$$

where

$$
\begin{aligned}
\bar{c}^{\mathcal{G}, \mathbf{d}} & =\frac{c^{\mathcal{G}, \mathbf{d}}}{\sum_{\mathbf{d} \in \mathcal{D}(\mathcal{G})} c^{\mathcal{G}, \mathbf{d}}} \\
\hat{\mathbf{x}}_{k \mid k}^{\mathcal{G}} & =\sum_{\mathbf{d} \in \mathcal{D}(\mathcal{G})} \bar{c}^{\mathcal{G}, \mathbf{d}_{\hat{\mathbf{x}}} \hat{\mathbf{G}}_{k \mid k}^{\mathcal{G}, \mathbf{d}}} \\
\hat{\mathbf{P}}_{k \mid k}^{\mathcal{G}} & =\sum_{\mathbf{d} \in \mathcal{D}(\mathcal{G})} \bar{c}^{\mathcal{G}, \mathbf{d}}\left\{\hat{\mathbf{P}}_{k \mid k}^{\mathcal{G}}+\left(\hat{\mathbf{x}}_{k \mid k}^{\mathcal{G}, \mathbf{d}}-\hat{\mathbf{x}}_{k \mid k}^{\mathcal{G}}\right)\left(\hat{\mathbf{x}}_{k \mid k}^{\mathcal{G}, \mathbf{d}}-\hat{\mathbf{x}}_{k \mid k}^{\mathcal{G}}\right)^{T}\right\} .
\end{aligned}
$$

\footnotetext{
${ }^{4}$ In fact, this the standard JPDA approximation performed for each resolution graph.
} 
TABLE II

TWO-STEP JPDA FILTER WITH RESOLUTION MODEL

I: Generate all feasible graphs, $\mathcal{G}$.

II : For each graph, formulate all data association hypotheses, $\mathcal{D}(\mathcal{G})$

III: For each data association hypothesis, $\mathbf{d} \in \mathcal{D}(\mathcal{G})$

III-a) Calculate $\operatorname{Pr}\left\{\mathbf{d} \mid \mathcal{G}, \mathbf{x}_{k}\right\}$ according to (28)

III-b) Update the predicted density with information from measurements, according to (38) and (39), or by (48). This yields a scaled Gaussian $c^{\mathcal{G}, \mathbf{d}} \mathcal{N}\left(\mathbf{x}_{k} ; \hat{\mathbf{x}}_{k \mid k}^{\mathcal{G}, \mathbf{d}}, \mathbf{P}_{k \mid k}^{\mathcal{G}, \mathbf{d}}\right)$ with weight given by (84).

IV: Perform moment matching over the data association hypotheses, as described in (91)-(94). This gives a single Gaussian $\mathcal{N}\left(\mathbf{x}_{k} ; \hat{\mathbf{x}}_{k \mid k}^{\mathcal{G}}, \mathbf{P}_{k \mid k}^{\mathcal{G}}\right)$.

V: For each graph, perform an update with the resolution model. This yields a Gaussian mixture, $\sum_{u \in \mathcal{U}(\mathcal{G})} \sum_{\mathbf{d} \in \mathcal{D}(\mathcal{G})} c^{\mathcal{G}, u} \mathcal{N}\left(\mathbf{x}_{k} ; \hat{\mathbf{x}}_{k \mid k}^{\mathcal{G}, u}, \mathbf{P}_{k \mid k}^{\mathcal{G}, u}\right)$, with weights given by (95), and mean value and covariance matrix by (93) and (94).

VI: Approximate the Gaussian mixture in (97) with a single Gaussian, as described in (98)-(100).

Step V: resolution update for each graph: After the moment matching in Step IV, we have a single Gaussian for each graph. In Step V, the resolution model update is performed under each graph. This gives a Gaussian mixture

$$
\sum_{u \in \mathcal{U}(\mathcal{G})} \sum_{\mathbf{d} \in \mathcal{D}(\mathcal{G})} \bar{c}^{\mathcal{G}, u} \mathcal{N}\left(\mathbf{x}_{k} ; \hat{\mathbf{x}}_{k \mid k}^{\mathcal{G}, u}, \mathbf{P}_{k \mid k}^{\mathcal{V}, u}\right)
$$

with weights

$$
\begin{gathered}
\bar{c}^{\mathcal{G}, u}=\frac{c^{\mathcal{G}, u}}{\sum_{u \in \mathcal{U}(\mathcal{G})} \sum_{\mathbf{d} \in \mathcal{D}(\mathcal{G})} c^{\mathcal{G}, u}}, \\
c^{\mathcal{G}, u}=\left|2 \pi \mathbf{R}_{u, N_{\text {res }}}\right| \mathcal{N}\left(\mathbf{0} ;\left(\mathbf{D}^{u} \otimes \mathbf{I}_{N_{\mathrm{res}}}\right) \tilde{\mathbf{H}} \hat{\mathbf{x}}_{k \mid k}^{\mathcal{G}, u}, \mathbf{S}^{\mathcal{G}, u}\right),
\end{gathered}
$$

where $\mathbf{S}^{\mathcal{G}, u}$ is given by (80), inserting $\mathbf{P}_{k \mid k}^{\mathcal{G}}$ instead of $\mathbf{P}_{k \mid k}$.

Step VI: Gaussian approximation of posterior density: For each graph, $\mathcal{G}$, Step V provides a Gaussian mixture. The posterior density is hence approximated by

$$
p\left(\mathbf{x}_{k} \mid \mathbf{Z}^{k}\right) \approx \sum_{\mathcal{G}} \sum_{u \in \mathcal{U}(\mathcal{G})} \bar{c}^{\mathcal{G}, u} \mathcal{N}\left(\mathbf{x}_{k} ; \hat{\mathbf{x}}_{k \mid k}^{\mathcal{G}, u}, \mathbf{P}_{k \mid k}^{\mathcal{G}, u}\right) .
$$


The final step of the algorithm is to make a second moment matching, to approximate the posterior density as a single Gaussian, according to

$$
p\left(\mathbf{x}_{k} \mid \mathbf{Z}^{k}\right) \approx \mathcal{N}\left(\mathbf{x}_{k} ; \hat{\mathbf{x}}_{k \mid k}^{A_{2}}, \mathbf{P}_{k \mid k}^{A_{2}}\right)
$$

where

$$
\begin{aligned}
\hat{\mathbf{x}}_{k \mid k}^{A_{2}} & =\sum_{\mathcal{G}} \sum_{u \in \mathcal{U}(\mathcal{G})} \bar{c}^{\mathcal{G}, u} \hat{\mathbf{x}}_{k \mid k}^{\mathcal{G}, u} \\
\mathbf{P}_{k \mid k}^{A_{2}} & =\sum_{\mathcal{G}} \sum_{u \in \mathcal{U}(\mathcal{G})} \bar{c}^{\mathcal{G}, u}\left\{\mathbf{P}_{k \mid k}^{\mathcal{G}, u}+\left(\hat{\mathbf{x}}_{k \mid k}^{\mathcal{G}, u}-\hat{\mathbf{x}}_{k \mid k}^{A_{2}}\right)\left(\hat{\mathbf{x}}_{k \mid k}^{\mathcal{G}, u}-\hat{\mathbf{x}}_{k \mid k}^{A_{2}}\right)^{T}\right\} .
\end{aligned}
$$

\section{Simulations}

In this section, we evaluate the JPDA filter with the proposed resolution model, and compare the results to those of the JPDA filter without a resolution model. The considered tracking scenario includes three targets whose trajectories are shown in Fig. 4. The targets move with a constant speed of $5 \mathrm{~m} / \mathrm{s}$, and their separation is $60 \mathrm{~m}$ in the middle part of the scenario.

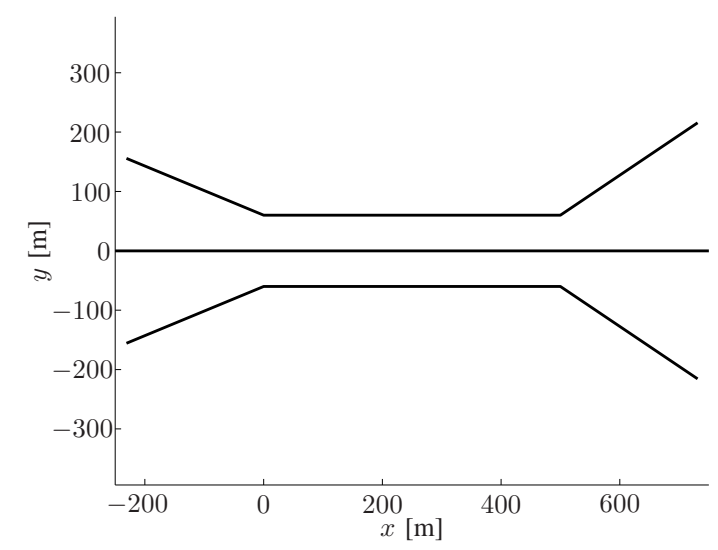

Fig. 4. Trajectories for the three-target scenario. The separation between the targets is $60 \mathrm{~m}$ in the middle, and the targets move from left to right.

As state vector, we use $\mathbf{x}=\left[\begin{array}{llll}x & y & \dot{x} & \dot{y}\end{array}\right]$. For prediction of future states in the filters, a (nearly) constant velocity model is used, with system matrix (cf. (4)) $\mathbf{F}=\operatorname{diag}\{\tilde{\mathbf{F}}, \tilde{\mathbf{F}}, \tilde{\mathbf{F}}\}$, where

$$
\tilde{\mathbf{F}}=\left[\begin{array}{cc}
\mathbf{I}_{2 \times 2} & T \cdot \mathbf{I}_{2 \times 2} \\
\mathbf{0}_{2 \times 2} & \mathbf{I}_{2 \times 2}
\end{array}\right],
$$


and where $T$ is the time between measurements. Further, the process noise covariance matrix is $\mathbf{Q}=$ $\operatorname{diag}\{\tilde{\mathbf{Q}}, \tilde{\mathbf{Q}}, \tilde{\mathbf{Q}}\}$, where

$$
\tilde{\mathbf{Q}}=q_{0}\left[\begin{array}{cc}
T^{3} / 3 \cdot \mathbf{I}_{2 \times 2} & T^{2} / 2 \cdot \mathbf{I}_{2 \times 2} \\
T^{2} / 2 \cdot \mathbf{I}_{2 \times 2} & T \cdot \mathbf{I}_{2 \times 2}
\end{array}\right],
$$

and $q_{0}=0.2$, which is the parameter value that gives the best average performance for this scenario.

At regular time intervals, $T$, of one second, a sensor, which is located at $(-10000,-10000)$, provides measurements of the range $R$ and azimuth angle $\varphi$ to the targets. The accuracy of the measurements depends on whether the targets are resolved or not, as discussed in Section IV. More specifically, the range errors are assumed to be constant as the number of targets in a group increases, while the azimuth errors are assumed to increase due to radar target glint. The target-number dependent measurement noise covariance matrix is thus

$$
\mathbf{R}^{n_{g}}=\operatorname{diag}\left\{\left(\sigma_{R}\right)^{2},\left(\sigma_{\varphi}^{n_{g}}\right)^{2}\right\}
$$

where $\sigma_{\varphi}^{n_{g}}=n_{g} \cdot 0.1 \pi / 180$. Further, the resolution capability of the radar sensor is given by the parameters (cf. (19)) $\alpha_{R}$ equal to $60 \mathrm{~m}$ and $\alpha_{\varphi}$ equal to $0.3 \pi / 180$. With these parameters, averaged over 100 trials for the 200 time steps of the scenario, all three targets are resolved 79.7 times (time steps), two targets are unresolved 70.7 times and all three targets form a group target 49.6 times.

The detection probability $P_{D}$ of the sensor is assumed equal for all group sizes. In the evaluations, we test detection probabilities of 0.999 and 0.95 . On top of target-generated measurements, the sensor also reports spurious measurements due to clutter. We evaluate the results for two different clutter levels: low clutter, with 1 false measurement per scan on average (and rarely more than 4), and moderate clutter, with 4 false measurements per scan on average (and rarely more than 8 ).

In Fig. 5, examples of trajectory estimates from the JPDA filter with and without the resolution model are shown, for the case of no clutter and a detection probability of 0.999. By comparing the outputs, it is seen that the filter which takes resolution limitations into account produces more stable, and wellseparated, tracks.

For single-target tracking, or tracking of widely separated targets, performance evaluation is straightforward. However, for tracking of densely-spaced targets, fair performance evaluation is a major concern, as noted already in [12]. There, a two-stage evaluation strategy was proposed, where first an optimal assignment of state estimates to ground truth is performed, and second a measure of choice (for example root mean squared error (RMSE)) is applied on that assignment. Recently, the Mean Optimal Subpattern Assignment (MOSPA) measure [21] was introduced, and evaluations based on that measure are increasing 

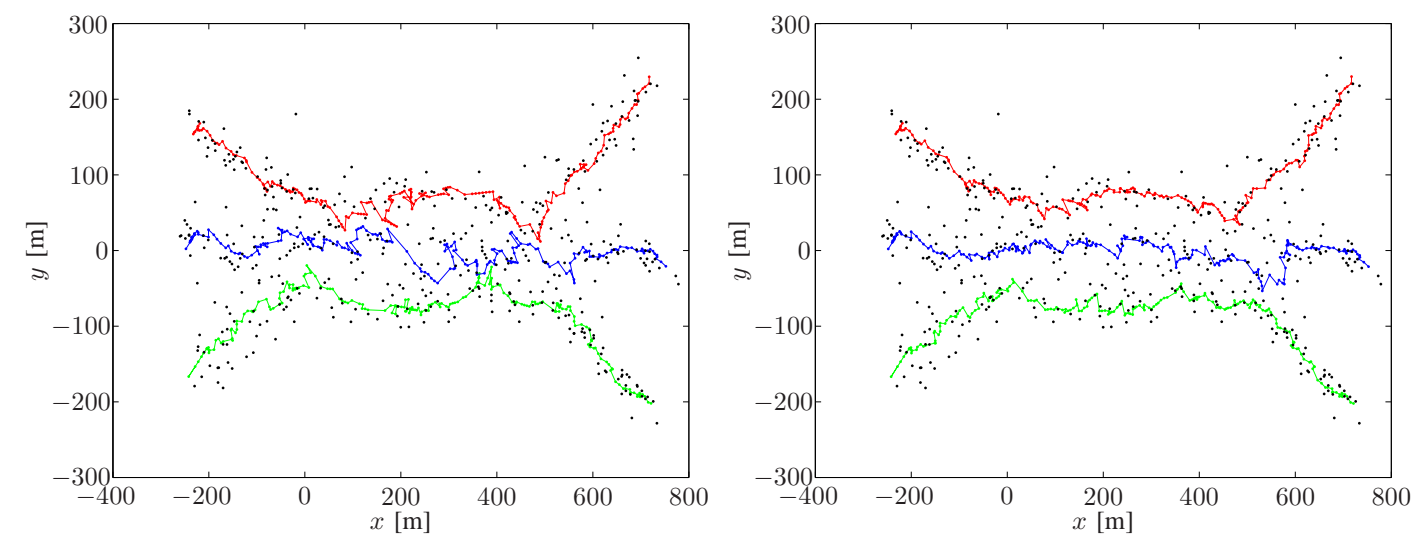

Fig. 5. Example of the output of the standard JPDA filter (left), and the full Gaussian mixture JPDA filter with a resolution model (right), for the case of no clutter and a detection probability of 0.999 , but with resolution limitations expressed by $\alpha_{R}=60 \mathrm{~m}$ and $\alpha_{\varphi}=0.3^{\circ}$. The dots represent the sensor measurements converted to Cartesian coordinates.

in number. The measure is a natural extension of the RMSE for multiple targets, and for a known number of targets it is equivalent to the procedure proposed in [12]. We have therefore used MOSPA in the evaluations of this article. As basis measure, we use the Euclidean distance. We further use the first-order MOSPA measure ( $p=1$, see [21]), which means that the unit of the MOSPA distance is meters. As cut-off value we use $c=300 \mathrm{~m}$.

For the performance evaluations, we first consider the case of a detection probability of 0.999 . To the left in Fig. 6, the MOSPA performance over 100 Monte Carlo runs is presented for the low-clutter case for the JPDA filters with and without a resolution model. As a reference, the performance of the JPDA filter when the sensor has perfect resolution is also shown. As seen, when the targets are closely spaced and the resolution limitations have effect, the JPDA filter without resolution model performs worse than the JPDA filter with a resolution model, which provides evidence that the resolution model indeed accounts for a better description of the data.

To the right in Fig. 6, the MOSPA results for the moderate clutter level are shown. There, it is seen that the JPDA filter performs significantly worse than with the lower clutter level, whereas the performance of the JPDA filter with a resolution model, performs almost as well. The reason for the significantly worse performance of the JPDA filter is that a large number of track losses occur (where tracks are attracted to clutter detections, and move away from the true trajectory). For the JPDA filter with a resolution model, on the other hand, the attraction of the clutter detections is weaker due to the filter considering the events that the targets are not resolved. 

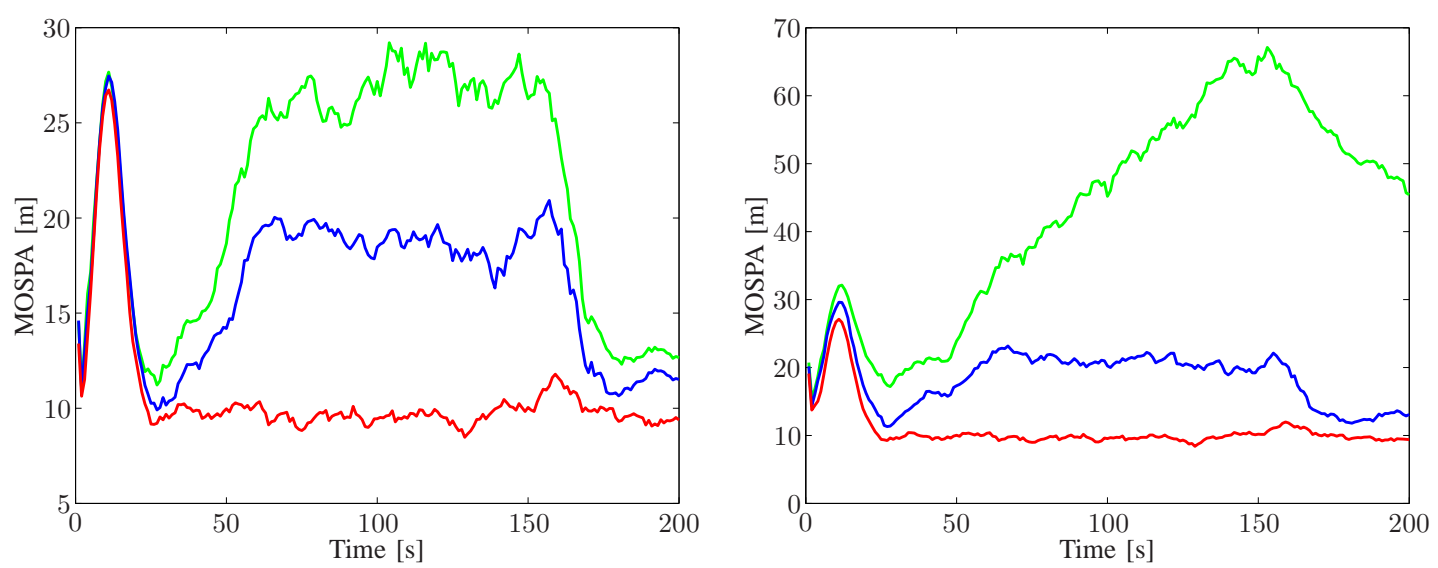

Fig. 6. MOSPA performance for the JPDA filters averaged over 100 Monte-Carlo runs for a detection probability of 0.999 . Left: one clutter measurement on average, right: four clutter measurements on average. Green: without resolution model, blue: with a resolution model, and red: with perfect resolution.

The final evaluation regards a lower detection probability of 0.95 , and a moderate clutter level. The results for this setup are shown in Fig. 7. By comparing this figure with the results shown to the right in Fig. 6, it is seen that the performance of the JPDA filter is somewhat better with the lower detection probability than with the higher one. The reason for this is that the weights of the data association hypotheses under which clutter detections are assigned to the tracks are lower when the detection probability is lower, and we thus obtain more stable tracks. Still, the performance is significantly improved by using the proposed sensor resolution model.

\section{CONCLUSION}

In this article, we consider the modeling of limited sensor resolution for an arbitrary, but known, number of targets. The main contribution is a framework for handling resolution limitations, which can be easily incorporated into traditional Bayesian tracking filters. The framework relies on a graph description of a resolution event, and on modeling the resolution probability as independent between target pairs. To complete the framework and to attain a multitarget resolution model, a model for the resolution probability for two targets and a group measurement model for an arbitrary number of targets are needed. By suggesting two such models, the exact calculation of the posterior probability density function under both data association and resolution conflicts is described. Under linear and Gaussian assumptions, the posterior density is a Gaussian mixture, and the components of that mixture are also derived in the paper. Further, two alternative approximations of the density mixture by a single Gaussian are proposed, 


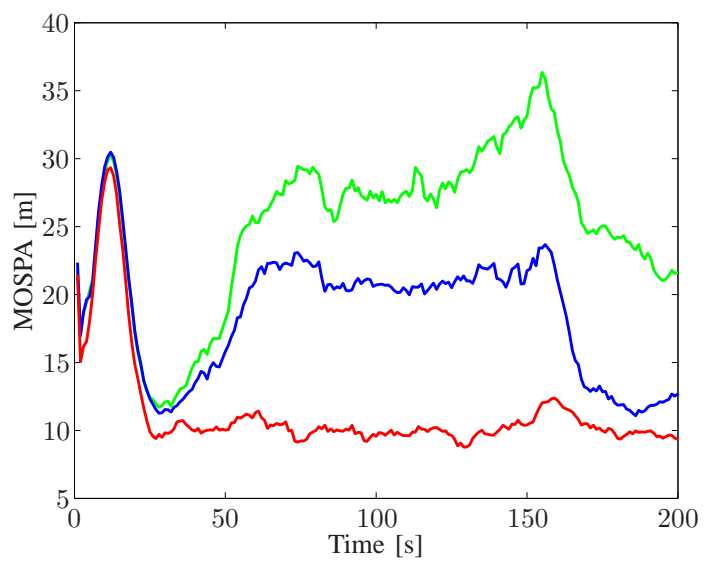

Fig. 7. MOSPA performance for the JPDA filters averaged over 100 Monte-Carlo runs for a detection probability of 0.95 and four clutter measurements on average. Green: without resolution model, blue: with a resolution model, and red: with perfect resolution.

which both can be considered as extensions of the Joint Probabilistic Data Association (JPDA) filter taking resolution problems into account. Finally, the JPDA filters with and without a resolution model are evaluated on a three-target tracking scenario with simulated radar data. The results show significantly improved tracking performance of the resolution filters for all considered setups.

\section{REFERENCES}

[1] S. Blackman, "Multiple hypothesis tracking for multiple target tracking," IEEE Aerospace and Electronic Systems Magazine, vol. 19, no. 1, pp. 5-18, January 2004.

[2] S. Blackman and R. Popoli, Design and Analysis of Modern Tracking Systems. Norwood, MA: Artech House, 1999.

[3] H. Blom and E. Bloem, "Exact bayesian filter and joint IMM coupled PDA tracking of maneuvering targets from possibly missing and false measurements," Automatica, vol. 42, pp. 127-135, 2006.

[4] — - "Joint particle filtering of multiple maneuvering targets from unassociated measurements," Journal of Advancement Information Fusion, vol. 1, pp. 15-36, 2006.

[5] _ - "Bayesian tracking of two possibly unresolved maneuvering targets," IEEE Transactions on Aerospace and Electronic Systems, vol. 43, no. 2, pp. 612-627, April 2007.

[6] — - "Hybrid SIR joint particle filtering under limited sensor resolution," in Proceedings of the 10th International Conference on Information Fusion, July 2007.

[7] J. Brewer, "Kronecker products and matrix calculus in system theory," IEEE Transactions on Circuits and Systems, vol. CAS-25, no. 9, pp. 772-781, September 1978.

[8] K. Chang and Y. Bar-Shalom, "Joint probabilistic data association with possibly unresolved measurements and maneuvers," IEEE Transactions on Automatic Control, vol. 29, no. 7, pp. 585-594, July 1984. 
[9] —_ "A simplification of the JPDAM algorithm," IEEE Transactions on Automatic Control, vol. 31, no. 10, pp. 989-981, October 1986.

[10] F. Daum and R. Fitzgerald, "Importance of resolution in multiple-target tracking," in Signal and Data Processing of Small Targets, ser. Proc. SPIE, vol. 2235, 1994.

[11] S. Deb, M. Yeddanapudi, K. Pattipati, and Y. Bar-Shalom, "A generalized s-d assignment algorithm for multisensormultitarget state estimation," IEEE Transactions on Aerospace and Electronic Systems, vol. 33, no. 2, pp. 523-538, April 1997.

[12] O. Drummond and B. Fridling, "Ambiguities in evaluating performance of multiple target tracking algorithms," in Signal and Data Processing of Small Targets, ser. Proc. SPIE, vol. 1698, 1992, pp. 326-337.

[13] T. Fortmann, Y. Bar-Shalom, and M. Scheffe, "Sonar tracking of multiple targets using joint probabilistic data association," IEEE Journal of Oceanic Engineering, vol. 8, no. 3, pp. 173-183, July 1983.

[14] J. Gunnarsson, L. Svensson, L. Danielsson, and F. Bengtsson, “Tracking vehicles using radar detections," in IEEE Intelligent Vehicles Symposium, 2007, pp. 296-302.

[15] A. Jazwinski, Stochastic Processes and Filtering Theory. New York: Academic, 1970, ch. 8, pp. $272-281$.

[16] S. Julier and J. Uhlmann, "Unscented Filtering and Nonlinear Estimation," Proceedings of the IEEE, vol. 92, no. 3, pp. 401-422, March 2004.

[17] R. Kalman, "A new approach to linear filtering and prediction problems," Transactions of the ASME-Journal of Basic Engineering, vol. 82 (Series D), pp. 35-45, 1960.

[18] W. Koch and G. van Keuk, "Multiple hypothesis track maintenance with possibly unresolved measurements," IEEE Transactions on Aerospace and Electronic Systems, vol. 33, no. 3, pp. 883-892, July 1997.

[19] S. Mori, K. Chang, and C. Chong, "Tracking aircraft by acoustic sensors-multiple hypothesis approach applied to possibly unresolved measurements," in American Control Conference, June 1987, pp. 1099-1105.

[20] D. Reid, “An algorithm for tracking multiple targets," IEEE Transactions on Automatic Control, vol. 24, no. 6, pp. 843-854, December 1979.

[21] D. Schuhmacher, B.-T. Vo, and B.-N. Vo, "A consistent metric for performance evaluation of multi-object filters," IEEE Transactions on Signal Processing, vol. 56, no. 8, pp. 3447-3457, August 2008.

[22] A. Sinha, T. Kirubarajan, and Y. Bar-Shalom, "Maximum likelihood angle extractor for two closely spaced targets," IEEE Transactions on Aerospace and Electronic Systems, vol. 38, no. 1, pp. 183-203, January 2002.

[23] _ _ "Tracker and signal processing for the benchmark problem with unresolved targets," IEEE Transactions on Aerospace and Electronic Systems, vol. 42, no. 1, pp. 279-300, January 2006.

[24] D. Svensson, M. Ulmke, and L. Danielsson, "Joint probabilistic data association filter for partially unresolved target groups," in Proceedings of the 13th International Conference on Information Fusion, July 2010.

[25] — - "Multitarget sensor resolution model for arbitrary target numbers," in Signal and Data Processing of Small Targets, ser. Proc. SPIE, vol. 7698, 2010.

[26] Z. Wang, A. Sinha, P. Willett, and Y. Bar-Shalom, "Angle estimation for two unresolved targets with monopulse radar," IEEE Transactions on Aerospace and Electronic Systems, vol. 40, no. 3, pp. 998-1019, July 2004.

[27] G. Watson and D. McCabe, "Benchmark problem with a multisensor framework for radar resource allocation and tracking of highly maneuvering targets, closely-spaced targets, and targets in the presence of sea-surface-induced multipath," NSWC Dahlgren, VA, USA, Tech. Rep. NSWC-DD/TR-99/32, March 1999. 
[28] M. Waxman and O. Drummond, “A bibliography of cluster (group) tracking," in Signal and Data Processing of Small Targets, ser. Proc. SPIE, vol. 5428, June 2004.

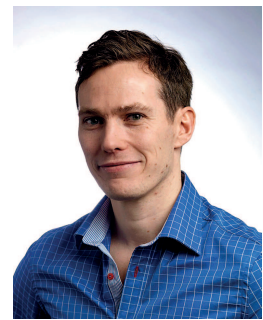

Daniel Svensson was born in Borås, Sweden in 1979. He received the M.Sc. degree in electrical engineering in 2004, and the Ph.D. degree in 2010, both from Chalmers University of Technology, Göteborg, Sweden.

Between 2004 and 2005, he was a Systems Engineer at Ericsson Microwave Systems, Göteborg, Sweden. Since November 2010 he is a Postdoctoral Research Fellow in the signal processing group at Chalmers University of Technology, and since April 2011 he is also a Systems Engineer at Electronic Defence Systems, Saab AB, Göteborg, Sweden. His research interests include nonlinear filtering, radar modelling and target tracking.

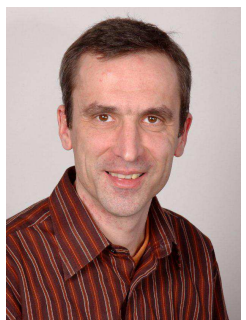

Martin Ulmke is theoretical physicist and received the Dipl. Phys. (M. S.) and Dr. rer. nat (Ph. D.) degrees from the Technical University in Aachen, Germany, in 1991 and 1995, respectively.

From 1995 to 1998 he was Research Associate in condensed matter theory at the University of California in Davis and at the University of Augsburg, Germany. He has been employed as Systems Engineer at MTU Aero Engines, Munich, from 1998 to 2001. Since 2001 he has been working in the Department "Sensor Data and Information Fusion" at Fraunhofer FKIE. His current research interests include estimation theory, Monte Carlo techniques, multiple target tracking, and ground surveillance.

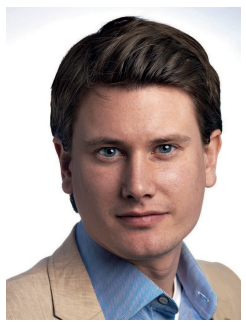

Lars Hammarstrand was born in Landvetter, Sweden in 1979. He received his M.Sc. and Ph.D. degrees in electrical engineering from Chalmers University of Technology, Gothenburg, Sweden, in 2004 and 2010, respectively.

From 2004 to 2011, he was with the Active Safety and Chassis Department at Volvo Car Corporation, Gothenburg, conducting research on tracking and sensor data fusion methods for active safety systems. Currently, he is a Postdoctoral Research Fellow at the Signal Processing group at Chalmers University of Technology where his main research interests are in the fields of estimation, sensor fusion and radar sensor modeling, especially with application to active safety systems. 